\title{
On the generalized Cauchy function and new Conjecture on its exterior singularities
}

\author{
Theodore Yaotsu Wu
}

Received: 6 January 2011 / Revised: 14 February 2011 / Accepted: 14 February 2011

(C)The Chinese Society of Theoretical and Applied Mechanics and Springer-Verlag Berlin Heidelberg 2011

\begin{abstract}
This article studies on Cauchy's function $f(z)$ and its integral, $(2 \pi \mathrm{i}) J[f(z)] \equiv \oint_{C} f(t) \mathrm{d} t /(t-z)$ taken along a closed simple contour $C$, in regard to their comprehensive properties over the entire $z=x+$ iy plane consisted of the simply connected open domain $\mathcal{D}^{+}$bounded by $C$ and the open domain $\mathcal{D}^{-}$outside $C$. (1) With $f(z)$ assumed to be $C^{n}$ $\left(n<\infty\right.$-times continuously differentiable) $\forall z \in \mathcal{D}^{+}$and in a neighborhood of $C, f(z)$ and its derivatives $f^{(n)}(z)$ are proved uniformly continuous in the closed domain $\overline{\mathcal{D}^{+}}=\left[\mathcal{D}^{+}+C\right]$. (2) Cauchy's integral formulas and their derivatives $\forall z \in \mathcal{D}^{+}$ (or $\forall z \in \mathcal{D}^{-}$) are proved to converge uniformly in $\overline{\mathcal{D}^{+}}$(or in $\left.\overline{\mathcal{D}^{-}}=\left[\mathcal{D}^{-}+C\right]\right)$, respectively, thereby rendering the integral formulas valid over the entire $z$-plane. (3) The same claims (as for $f(z)$ and $J[f(z)]$ ) are shown extended to hold for the complement function $F(z)$, defined to be $C^{n} \forall z \in \mathcal{D}^{-}$and about $C$. (4) The uniform convergence theorems for $f(z)$ and $F(z)$ shown for arbitrary contour $C$ are adapted to find special domains in the upper or lower half $z$-planes and those inside and outside the unit circle $|z|=1$ such that the four generalized Hilbert-type integral transforms are proved. (5) Further, the singularity distribution of $f(z)$ in $\mathcal{D}^{-}$is elucidated by considering the direct problem exemplified with several typical singularities prescribed in $\mathcal{D}^{-}$. (6) A comparative study is made between generalized integral formulas and Plemelj's formulas on their differing basic properties. (7) Physical significances of these formulas are illustrated with applications
\end{abstract}

\footnotetext{
Th. Y. Wu (凶)

California Institute of Technology, Pasadena, CA 91125 USA

Honorary Research Fellow, Institute of Mechanics,

Chinese Academy of Sciences

e-mail: tywu@caltech.edu
}

to nonlinear airfoil theory. (8) Finally, an unsolved inverse problem to determine all the singularities of Cauchy function $f(z)$ in domain $\mathcal{D}^{-}$, based on the continuous numerical value of $f(z) \forall z \in \overline{\mathcal{D}^{+}}=\left[\mathcal{D}^{+}+C\right]$, is presented for resolution as a conjecture.

Keywords Uniform continuity of Cauchy's function · Uniform convergence of Cauchy's integral formula · Generalized Hilbert-type integral transforms - Functional properties and singularity distributions

\section{Introduction}

This article is a special final version of $\mathrm{Wu}(2007)$ [1]. In the classical studies of water waves propagating in permanent form on water of finite or infinite depth in the physical $z=x+$ iy plane, the complex potential $f=\phi+\mathrm{i} \psi$ was first adopted by Stokes (1880) [2] as the independent variable for the advantage of its boundary location being known. The infinite strip of $f$ for a solitary wave or a semi-infinite strip of $f$ for a periodic wave train on deep water can be mapped conformally onto a unit disc $(|\zeta| \leq 1)$ in the $\zeta=\xi+\mathrm{i} \eta$ plane. For waves of a rounded crest, the complex velocity $w(z(\zeta))=\mathrm{d} f / \mathrm{d} z$ is an analytic function, regular (or holomorphic, i.e. one-valued and continuously differentiable) in the closed flow domain. As the solitary, or a simple periodic wave (of amplitude $a$ and wave number $k$ ) grows to become the highest with a corner crest of interior angle of $120^{\circ}, w(z(\zeta))$ then has a primary (Stokes's) and secondary (Grant's) algebraic branch singularities at the crest on the flow boundary $|\zeta|=1$, whereas $w(z(\zeta))$ remains regular in 
the open interior domain $|\zeta|<1$. This will be taken as the direct problem of permanent water waves.

A foremost query is to question: "Where do the branch singularities go as the highest wave becomes rounded at the crest by a slight reduction in height?" "Do the singularities retain their type, with only changes in strength and location to an interior point in the open domain $\mathcal{D}^{-}\{\forall \zeta \in|\zeta|>1\}$ outside the unit disc of the flow domain? or even with changes in types of singularities?" In fact, for waves that are regular in the closed flow domain $\overline{\mathcal{D}^{+}}\{\forall \zeta \in|\zeta| \leq 1\}, w(z(\zeta))$ must have singularities distributed in the open domain $\mathcal{D}^{-}$, for otherwise, by Liouville's Theorem, $w(z(\zeta))$ would have to be a constant in the entire $\zeta$-plane, which is the trivial case of a wave of vanishing amplitude.

To determine the singularities of $w(z(\zeta))$ in domain $\mathcal{D}^{-}$ outside the flow field based on solutions obtained in numerics, to be called the inverse problem in short, is of fundamental importance to gaining sound physical and mathematical expositions to a growing list of intriguing and even perplexing properties of these waves as exhibited in their solutions. In particular, this need has been motivated by a recent series of studies. First, the perturbation expansion method introduced by Stokes (1847) [2] for studies on the nonlinear and dispersive effects in water waves by means of series solution in powers of certain small parameters, and followed by various authors in the active history of solitary waves, is still to be concluded on the convergence, or not, of such power series expansion, as reviewed by $\mathrm{Wu}$ et al. [3], who have brought the one-parameter power series for solitary waves (of height $a$ and wave-number $k$ in water of depth $h$ ) to the eighteenth order, finding the series to become asymptotic, reaching a minimum error at order $n=12$ if parametric in $\alpha=a / h$, and at order $n=17$ if in $\epsilon=k^{2} h^{2}$.

In this premise, $\mathrm{Wu}$ et al. [4] have developed a unified intrinsic functional expansion theory for exact evaluation on the Euler model for solitary waves of arbitrary specific height $\alpha=a / h$. This theory adopts an expansion in terms of a set of intrinsic component functions determined from analysis of the flow field about the wave crest, its outskirts, and its mid-spans, with the unknown coefficients determined by minimizing the mean-square-error of the Bernoulli constant $(=0)$. For the highest wave with a corner crest of $120^{\circ}$, taking 15 unknown coefficients of the series expansion optimally selected has yielded results including $\alpha=a / h=0.8331990$ for the height, the Froude number $F=c / \sqrt{g h}=1.2908904$ for the wave speed $c$, accurate to six decimals. Comparable accuracies have been attained [4] uniformly for solitary waves of all heights down to the so-called dwarf long waves defined for waves of amplitude $\alpha=a / h<10^{-2}$, including tsunamis commonly assessed as of amplitude $\alpha=O\left(10^{-3}-10^{-4}\right)$ in the open ocean. This subject is still an open field in view of the findings [4] that the relative errors of the solution spread out many waterdepths farther off, the lower the wave. It can be expected that these challenging issues could be overcome once the singu- larity distributions of the solution variable outside the flow field be determined by a general theoretical method. It may further expose the mechanisms underlying wave instabilities, or bifurcation of any steady-state solution found. All such pursuits could benefit by the inverse problem resolved. This is the principal objective of the present study.

\section{The Cauchy function with Cauchy's integral theorem and integral formulas}

For the general purpose, we first re-cite the two coupled Cauchy's integral formulas, known as the founding stones of the theory of functions of a complex variable $z=x+\mathrm{i} y$,

$$
\begin{aligned}
J[f(z)] & \equiv \frac{1}{2 \pi \mathrm{i}} \oint_{C} \frac{f(t)}{t-z} \mathrm{~d} t=f(z), \\
(z & \left.\in \mathcal{D}^{+}-\text {open domain inside } C,|z|<1\right), \\
J[f(z)] & \equiv \frac{1}{2 \pi \mathrm{i}} \oint_{C} \frac{f(t)}{t-z} \mathrm{~d} t=0, \\
(z & \left.\in \mathcal{D}^{-} \text {- open domain outside } C,|z|>1\right),
\end{aligned}
$$

where $C$ is a closed contour, say a unit circle: $|z|=|t|=$ $1 \forall t \in C$ traversing in +ive (counter-clockwise) sense as the standard reference, and here $f(z)$ is defined as the Cauchy function. This and the following two Theorems can be found in Titchmarsh (1949) [5] and other literature to which we refer.

Definition 1. The Cauchy function. If $f(z)$ is analytic and regular in a simply-connected open domain $\mathcal{D}^{+}$bounded by contour $C$ and if $\mathrm{d} f / \mathrm{d} z$ is continuous on $C, f(z)$ is a Cauchy function. Domain $\mathcal{D}^{+}$plus contour $C$ plus the open domain $\mathcal{D}^{-}$outside $C$ (including $z=\infty$ ), i.e. $\left[\mathcal{D}^{+}+C+\mathcal{D}^{-}\right]$, constitutes the entire $z$-plane. With the Cauchy function there are several related classical theorems.

Theorem 1. The Cauchy-Riemann equations. If $f(z)$ is analytic, meaning it is continuously differentiable $\forall z \in$ domain $\mathcal{D}, f(z)=u(x, y)+\mathrm{i} v(x, y)$ has its conjugate functions $u(x, y)$ and $v(x, y)$ satisfy in $\mathcal{D}$ the relations

$\frac{\partial u}{\partial x}=\frac{\partial v}{\partial y}, \quad \frac{\partial u}{\partial y}=-\frac{\partial v}{\partial x}$,

(the Cauchy-Riemann equations).

Theorem 2. Cauchy's integral theorem. If $f(z)$ is analytic and regular (finite and one-valued) in a simply-connected domain $\mathcal{D}^{+}$inside and on contour $C$, then the integral,

$\int_{z_{0}}^{z} f(z) \mathrm{d} z=F(z)-F\left(z_{0}\right), \quad(\mathrm{d} F(z) / \mathrm{d} z=f(z))$,

depends only on the end points $z_{0}$ and $z$, but not on the path between them in $\mathcal{D}^{+}$, hence equivalently,

$I[f(z)] \equiv \oint_{C} f(z) \mathrm{d} z=0$.

In fact, Eq. (3b) follows from Eq. (2) since $f(z) \mathrm{d} z=$ 
$(u \mathrm{~d} x-v \mathrm{~d} y)+\mathrm{i}(v \mathrm{~d} x+u \mathrm{~d} y)$, both terms being integrable.

Returning to Eq. (1), Eq. (1b) directly follows from Eq. (3b), for $g(t, z)=f(t) /(t-z)$ is regular $\forall t \in C$ and $\forall z \in \mathcal{D}^{-}$, and Eq. (1a) follows from the residue theorem.

Here, the Cauchy integral, denoted by $J[f(z)]$, also to be called the Cauchy functional (bearing an integral operator connotation), possesses its value equal to $f(z) \forall z \in \mathcal{D}^{+}$inside $C$ and vanishes identically $\forall z \in \mathcal{D}^{-}$outside $C$, all in terms of its values $f(t)$ on $C$. But the most obvious gap between Eqs. (1a) and (1b) is the conspicuous lack of a value for $J[f(z)]$ when $z$ falls right on contour $C$. To have all the properties of $f(z)$ thoroughly expounded, the primary objective of the present study is to fill this long-standing gap and have the two integral formulas cover the entire $z=x+\mathrm{i} y$ plane.

To proceed, we first extend the original assumption invoked on the Cauchy function $f(z)$.

\section{Generalization under new assumption}

We first introduce a new assumption that $f(z) \in C^{n}(n<\infty)$ $\forall z \in \mathcal{D}^{+}$and in a neighborhood striding across contour $C$.

Next, we conduct a limiting procedure to let a point $z_{+} \in \mathcal{D}^{+}$ in Eq. (1a) and a point $z_{-} \in \mathcal{D}^{-}$in Eq. (1b) each tend from the $\mathcal{D}^{ \pm}$side, respectively, to a common generic point $z_{0} \in C$ by following two principles:

(1) Contour $C$ is deformed into $C^{ \pm}$with a small semicircle $C_{\epsilon}^{ \pm}$of radius $\epsilon$ centered at $z_{0} \in C$ and indented onto the $\mathcal{D}^{\mp}$ side to let point $z_{ \pm}\left(\in \mathcal{D}^{ \pm}\right)$reach $z_{0}$ without crossing $C^{ \pm}$, respectively.

(2) The value of Cauchy's integral remains intact in the limit as $\epsilon \rightarrow 0$ and $z_{ \pm} \rightarrow z_{0}$ without crossing either $C^{+}$by $z_{+}$or $C^{-}$by $z_{-}$.

Definition 2. Generalized Cauchy function. The Cauchy function as just specified under the new assumption will be called the generalized Cauchy function for identifying its discreet distinction.

\subsection{Uniform continuity of the generalized Cauchy func- tion $f(z)$}

First, we let point $z_{ \pm} \in \mathcal{D}^{ \pm}$tend, respectively, to a generic point $z_{0}$ on $C$ just as specified by Principle (1), and hence Principle (2) is fulfilled (as $z_{ \pm}$reach $z_{0} \in C$ without crossing $C^{ \pm}$) while $C_{p}=C-C_{\epsilon}^{ \pm}$remains intact (see Fig. 1). Further, with the contour so deformed, Principle (2) is clearly observed. Hence

$$
\begin{aligned}
I^{ \pm}\left(z_{0}\right) & \equiv \oint_{C^{ \pm}} \frac{f(t)}{t-z_{0}} \mathrm{~d} t \\
& =\left\{\int_{C_{\epsilon}^{ \pm}}+\int_{C_{p}}\right\} \frac{f(t)}{t-z_{0}} \mathrm{~d} t \\
& =I_{\epsilon}^{ \pm}\left(z_{0}\right)+I_{p}\left(z_{0}\right), \quad(\epsilon>0), \\
I_{\epsilon}^{ \pm}\left(z_{0}\right) & =f\left(z_{0}\right) \int_{C_{\epsilon}^{ \pm}} \frac{\mathrm{d} t}{t-z_{0}}+J\left(z_{0}\right) \rightarrow \pm \pi \mathrm{i} f\left(z_{0}\right), \\
J\left(z_{0}\right) & =\int_{C_{\epsilon}^{ \pm}} \frac{f(t)-f\left(z_{0}\right)}{t-z_{0}} \mathrm{~d} t \rightarrow 0, \quad(\epsilon \rightarrow 0),
\end{aligned}
$$

where the limit for $I_{\epsilon}^{ \pm}\left(z_{0}\right)$ comes with $t$ moving in the \pm sense on $C_{\epsilon}^{ \pm}$, whilst that for $J\left(z_{0}\right)$ results from the argument that for given $\epsilon>0, \exists \delta\left(\epsilon, z_{0}\right) \ni\left|\left(f(t)-f\left(z_{0}\right)\right) /\left(t-z_{0}\right)-f^{\prime}\left(z_{0}\right)\right|<\delta$, $\forall\left|t-z_{0}\right|<\epsilon$, hence $\left|J\left(z_{0}\right)\right|<\left(\left|f^{\prime}\left(z_{0}\right)\right|+\delta\right) \ell_{\epsilon}\left(C_{\epsilon}^{ \pm}\right.$being of length $\left.\ell_{\epsilon}=\pi \epsilon\right) \rightarrow 0$ as $\epsilon \rightarrow 0$. In addition, as $\epsilon \rightarrow 0$, the integral over $C_{p}$ assumes Cauchy's principal value defined by

$$
\begin{aligned}
I_{p}\left(z_{0}\right) & =\int_{C_{p}} \frac{f(t)}{t-z_{0}} \mathrm{~d} t \rightarrow \lim _{\epsilon \rightarrow 0} \int_{z_{0}+\epsilon}^{z_{0}-\epsilon} \frac{f(t) \mathrm{d} t}{t-z_{0}} \\
& =\mathcal{P} \oint_{C} \frac{f(t) \mathrm{d} t}{t-z_{0}}, \quad\left(z_{0} \text { on } C\right),
\end{aligned}
$$

with $z_{0} \pm \epsilon$ both lying on $C$, and with the symbol $\mathcal{P}$ signifying Cauchy's principal value often omitted by convention as understood.
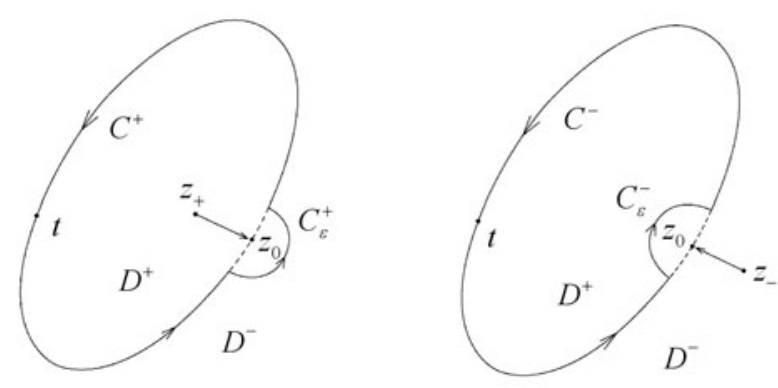

Fig. 1 A Cauchy integral $\oint_{C} f(t) \mathrm{d} t /(t-z)$ around a simple closed contour $C$ enclosing domain $\mathcal{D}^{+}$and excluding domain $\mathcal{D}^{-}$has its contour deformed into $C^{ \pm}$with only a small semicircle $C_{\epsilon}^{ \pm}$indented onto the $\mathcal{D}^{\mp}$ side, centered at $z_{0} \in C$ so as to let point $z_{ \pm} \in \mathcal{D}^{ \pm}$tend, respectively, to $z_{0} \in C$ without crossing $C^{ \pm}$

Finally, as $z_{+} \rightarrow z_{0}$ and $\epsilon \rightarrow 0$, Eq. (1a) yields $J\left[f\left(z_{0}\right)\right]=\left(I_{\epsilon}^{+}+I_{p}\right) /(2 \pi \mathrm{i}) \rightarrow f^{+}\left(z_{0}\right)$, a limiting value of $f(z)$, yet to be determined. And as $z_{-} \rightarrow z_{0}, \epsilon \rightarrow 0$, Eq. (1b) gives $J\left[f\left(z_{0}\right)\right]=\left(I_{\epsilon}^{-}+I_{p}\right) /(2 \pi \mathrm{i}) \rightarrow 0$, since $J\left[f\left(z_{0}\right)\right]$ of Eq. (1b) remains zero by Principle (2). Thus, Eq. (1) become

$$
\begin{aligned}
f^{+}\left(z_{0}\right) & =\lim _{z_{+} \rightarrow z_{0}} \frac{1}{2 \pi \mathrm{i}} \oint_{C} \frac{f(t)}{t-z_{+}} \mathrm{d} t \\
& =\frac{1}{2} f\left(z_{0}\right)+\frac{1}{2 \pi \mathrm{i}} \mathcal{P} \oint_{C} \frac{f(t)}{t-z_{0}} \mathrm{~d} t, \quad\left(z_{0} \text { on } C\right),
\end{aligned}
$$




$$
\begin{aligned}
0 & =\lim _{z_{-} \rightarrow z_{0}} \frac{1}{2 \pi \mathrm{i}} \oint_{C} \frac{f(t)}{t-z_{-}} \mathrm{d} t \\
& =-\frac{1}{2} f\left(z_{0}\right)+\frac{1}{2 \pi \mathrm{i}} \mathcal{P} \oint_{C} \frac{f(t)}{t-z_{0}} \mathrm{~d} t, \quad\left(z_{0} \text { on } C\right),
\end{aligned}
$$

of which the sum and difference determine two relations of vital importance as

$$
\begin{aligned}
& \text { (I) : } f^{+}(z)=f(z) ; \\
& \text { (II) : } f(z)=\frac{1}{\pi \mathrm{i}} \mathcal{P} \oint_{C} \frac{f(t)}{t-z} \mathrm{~d} t, \quad(z \text { on } C),
\end{aligned}
$$

in which the suffix of $z_{0}$ is omitted for all $z$ on $C$. Here, the first relation (I), $f^{+}(z)=f(z)$, shows that the limit $f^{+}(z)$ of $f(z)$ reached from the $\mathcal{D}^{+}$side is equal to the original $f(z)$ prescribed $\forall z \in C$; it therefore proves the uniform continuity of $f(z)$ in the closed domain $\overline{\mathcal{D}^{+}}=\left[\mathcal{D}^{+}+C\right]$. Relation (II) gives the value to $f(z)$ for any $z \in C$ in terms of its values $f(t)$ over $C$. The integral representing $f(z)$ for $z \in C$ in Relation (II) is noted to differ from that of $f(z)$ for $z \notin C$ in Eq. (1a) by a factor of 2 , with the new integral assuming its principal value. This completes the proof of the above two key relations in Eq. (6) by finding formula (6a), (6b) as the limit values of $J[f(z)]$ of both Eq. (1a) and Eq. (1b), respectively. By Eq. (6c), we therefore have proved.

Theorem 3. Uniform continuity of generalized Cauchy function $f(z)$ in closed domain $\overline{\mathcal{D}^{+}}$. If $f(z)$ satisfies Cauchy's integral formula (1a) in open domain $\mathcal{D}^{+}$bounded by contour $C$ and also Eq. (1b) in open domain $\mathcal{D}^{-}$outside $C$, then $f(z)$ is uniformly continuous within the closed domain $\overline{\mathcal{D}^{+}}=\left[\mathcal{D}^{+}+C\right]$.

\subsection{Uniform convergence of the integral formulas and re- sulting integral properties}

From Theorem 3 there readily follow other important consequences:

Theorem 4. Uniform convergence of the integral formulas. The integral formulas (1) for $J[f(z)] \forall z \in \mathcal{D}^{+}$(or $\forall z \in \mathcal{D}^{-}$) converge uniformly in closed domain $\overline{\mathcal{D}^{+}}$(or $\left.\overline{\mathcal{D}^{-}}\right)$, respectively.

Proof: Rewrite Eq. (1) as

$$
\begin{aligned}
& g(z) \equiv J[f(z)]-f(z)=0, \quad\left(z \in \mathcal{D}^{+}\right), \\
& G(z) \equiv J[f(z)]=0, \quad\left(z \in \mathcal{D}^{-}\right) .
\end{aligned}
$$

In Eq. (7a), let $z_{ \pm} \in \mathcal{D}^{ \pm}$tend, respectively, to a generic point $z_{0} \in C$, with contour $C$ indented into $C_{ \pm}$as just delineated (see Fig. 1), thus yielding Eq. (6) for the values of the integral $J[f(z)]$ in the limit. These two limiting equations, upon using the two relations in Eq. (6c), then both become

$$
\begin{aligned}
g\left(z_{0}\right) & =G\left(z_{0}\right) \\
& =-\frac{1}{2} f\left(z_{0}\right)+\frac{1}{2 \pi \mathrm{i}} \mathcal{P} \oint_{C} \frac{f(t)}{t-z_{0}} \mathrm{~d} t=0, \quad\left(z_{0} \in C\right) .
\end{aligned}
$$

Therefore, Eqs. (7a) and (7b) state that for $z_{0} \in C$,

$$
\begin{aligned}
& g(z)=J[f(z)]-f(z)=g\left(z_{0}\right)=0, \quad\left(z \in \overline{\mathcal{D}^{+}}\right) ; \\
& G(z) \equiv J[f(z)]=G\left(z_{0}\right)=0, \quad\left(z \in \overline{\mathcal{D}^{-}}\right),
\end{aligned}
$$

which clearly proves the uniform convergence of $g(z)$ in closed domain $\overline{\mathcal{D}^{+}}$and the uniform convergence of $G(z)$ in closed domain $\overline{\mathcal{D}^{-}}=\left[\mathcal{D}^{-}+C^{-}\right]$, as was to prove.

\subsection{Derivatives of Cauchy's integral formulas}

It is classical [5] that Cauchy's integral $J[f(z)]$ has derivatives $J_{n}[f(z)]$ of all orders, founded on the same basis as that for Eq. (1), given by

$$
\begin{gathered}
J_{n}[f(z)] \equiv \frac{\mathrm{d}^{n}}{\mathrm{~d} z^{n}} J[f(z)]=\frac{n !}{2 \pi \mathrm{i}} \oint_{C} \frac{f(t) \mathrm{d} t}{(t-z)^{n+1}}=f^{(n)}(z), \\
\left(z \in \mathcal{D}^{+} ; n=1,2, \cdots\right), \\
J_{n}[f(z)] \equiv \frac{\mathrm{d}^{n}}{\mathrm{~d} z^{n}} J[f(z)]=\frac{n !}{2 \pi \mathrm{i}} \oint_{C} \frac{f(t) \mathrm{d} t}{(t-z)^{n+1}}=0, \\
\left(z \in \mathcal{D}^{-} ; n=1,2, \cdots\right) .
\end{gathered}
$$

Now, we can also attain Eq. (9) by differentiation under the integral sign of Eq. (1), by virtue of Theorem 4.

Further, integrating the integral in Eq. (9) by parts $m(\leq n)$ times yields

$$
\begin{gathered}
J_{(n, m)}[f(z)] \equiv \frac{(n-m) !}{2 \pi \mathrm{i}} \oint_{C} \frac{f^{(m)}(t) \mathrm{d} t}{(t-z)^{n-m+1}}=f^{(n)}(z), \\
\left(z \in \mathcal{D}^{+} ; m=0,1, \cdots, n\right), \\
J_{(n, m)}[f(z)] \equiv \frac{(n-m) !}{2 \pi \mathrm{i}} \oint_{C} \frac{f^{(m)}(t) \mathrm{d} t}{(t-z)^{n-m+1}}=0, \\
\left(z \in \mathcal{D}^{-} ; n=1,2, \cdots\right),
\end{gathered}
$$

in which every integrated term in each step vanishes under the new assumption. These $J_{(n, m)}[f(z)]$ are $m$ equivalent integral forms for $f^{(n)}(z)$. In particular, for $m=n, J_{(n, n)}[f(z)] \equiv$ $J_{n}[f(z)]$, we obtain

$$
\begin{gathered}
J_{n}[f(z)]=\frac{1}{2 \pi \mathrm{i}} \oint_{C} \frac{f^{(n)}(t)}{t-z} \mathrm{~d} t=f^{(n)}(z), \\
\left(z \in \mathcal{D}^{+} ; n=1,2, \cdots\right), \\
J_{n}[f(z)]=\frac{1}{2 \pi \mathrm{i}} \oint_{C} \frac{f^{(n)}(t)}{t-z} \mathrm{~d} t=0, \\
\left(z \in \mathcal{D}^{-} ; n=1,2, \cdots\right) .
\end{gathered}
$$

This new result for the generalized function $f(z)$ is very valuable, for writing $h(z)=f^{(n)}(z)$ renders Eq. (11) identical in form with Eq. (1), and can therefore be uniformly treated all together. Whence,

Theorem 5. Uniform continuity of the derivatives $f^{(n)}(z)$ in closed domain $\overline{\mathcal{D}^{+}}$. If $f(z)$ satisfies Eq. (1a) in open domain $\mathcal{D}^{+}$and also Eq. (1b) in open domain $\mathcal{D}^{-}$, and if $f^{(n)}(z)$ is continuous on $C$ as assumed, then $f^{(n)}(z)$ is uniformly continuous within the closed domain $\overline{\mathcal{D}^{+}}=\left[\mathcal{D}^{+}+C\right]$, with 


$$
\begin{aligned}
& f^{(n)+}(z)=f^{(n)}(z) ; \\
& f^{(n)}(z)=\frac{1}{\pi \mathrm{i}} \mathcal{P} \oint_{C} \frac{f^{(n)}(t)}{t-z} \mathrm{~d} t, \quad(z \text { on } C ; n=1,2, \cdots) .
\end{aligned}
$$

In fact, this follows in complete analogy with Eq. (6) since Eq. (11) for $J_{n}[f(z)]$ are identical in form with Eq. (1) for $J[f(z)]$. By the same analogy with Eq. (8) in Theorem 4, we also have the important consequence.

Theorem 6. Uniform convergence of formulas of $J_{n}[f(z)]$. The integral formulas of $J_{n}[f(z)] \forall z \in \mathcal{D}^{+}\left(\right.$or $\left.\forall z \in \mathcal{D}^{-}\right)$converge uniformly in closed domain $\overline{\mathcal{D}^{+}}\left(\right.$or $\left.\overline{\mathcal{D}^{-}}\right)$, respectively, for $n=0,1, \cdots$,

$$
\begin{aligned}
& g_{n}(z)=J_{n}[f(z)]-f^{(n)}(z)=g_{n}\left(z_{0}\right)=0, \quad\left(z \in \overline{\mathcal{D}^{+}}\right) \\
& G_{n}(z)=J_{n}[f(z)]=G_{n}\left(z_{0}\right)=0, \quad\left(z \in \overline{\mathcal{D}^{-}}\right) .
\end{aligned}
$$

For further studies in the sequel, we chart out the following course.

A new complement function $F(z)$ is introduced to be $C^{n} \forall z \in \mathcal{D}^{-}$outside $C$ and about $C$, and shown to share all the claims for $f(z)$ in complete analogy. In Sect. 5, the uniform continuity found for generalized function $f(z)$ in domain $\overline{\mathcal{D}^{+}}$ and also the complement function $F(z)$ in $\overline{\mathcal{D}^{-}}=\left[\mathcal{D}^{-}+C\right]$ are jointly adapted and applied to special domains in the upper-, or lower-half $z$-plane and those inside or outside the unit circle $|z|=1$ to have the generalized Hilbert transforms proved for these cases.

The central point is stressed that the generalized function $f(z)$ itself can have such singularity distributions in $\mathcal{D}^{-}$ as dictated only by its numerical values $f(t) \forall t \in C$. Possible behavior of function $f(z)$ in $\mathcal{D}^{-}$is illustrated in Sect. 7 as a direct problem (to which our inverse problem is its inverse) with singularities exemplified by poles, algebraic and logarithmic branches directly prescribed in $\mathcal{D}^{-}$.

This study is extended in Sect. 7 and Sect. 8 to include Plemelj's formulas for a line integral of a regular function along a Jordan arc without a double point (not closed as a contour) for applications to nonlinear wing theory. It is concluded in Sect. 9 with expository discussions for further studies. Especially, the inverse problem to determine analytically the singularity distribution of function $f(z)$ in open domain $\mathcal{D}^{-}$outside contour $C$ in terms of its numerical values on $C$ is presented for resolution as a conjecture.

\subsection{Extension to domains of other forms}

The original simply-connected open domain $\mathcal{D}^{+}$bounded by arbitrary contour $C$ can be extended to domains of other geometric forms. For simplicity, contour $C$ will still assume the unit circle in this section.

Complement function. Interchanging the roles of $\mathcal{D}^{ \pm}$gives rise to the complement function, denoted by $F(z)$, defined in complete analogy with function $f(z)$, i.e. being $C^{n} \forall z \in \mathcal{D}^{-}$ and in a neighborhood striding across contour $C$, includ- ing $z=\infty$ such that $F(z)=O\left(|z|^{-m}\right)(m \geq 2)$ as $|z| \rightarrow \infty$. The analogous integral of $F(z)$, denoted by $J^{-}[F(z)]$, will be called the complement functional.

Complement function $F(z)$ satisfies the integral theorem $3 \forall z \in \mathcal{D}^{-}$and its integral formulas

$$
\begin{aligned}
J^{-}[F(z)] & \equiv \frac{1}{2 \pi \mathrm{i}} \oint_{C^{-}} \frac{F(t)}{t-z} \mathrm{~d} t \\
& =\frac{-1}{2 \pi \mathrm{i}} \oint_{C} \frac{F(t)}{t-z} \mathrm{~d} t=F(z), \quad\left(z \in \mathcal{D}^{-}\right), \\
J^{-}[F(z)] & \equiv \frac{1}{2 \pi \mathrm{i}} \oint_{C^{-}} \frac{F(t)}{t-z} \mathrm{~d} t \\
& =\frac{-1}{2 \pi \mathrm{i}} \oint_{C} \frac{F(t)}{t-z} \mathrm{~d} t=0, \quad\left(z \in \mathcal{D}^{+}\right),
\end{aligned}
$$

in which the negative sign for the integral around $C$ is due to the positive sense of $C^{-}$being clockwise, opposite in sense to $C$. This can also be derived by applying the inverse conformal map $z=1 / \zeta, t=1 / \tau$ about the unit circle of $C$, mapping $C$ onto $C^{*}$ (also taken counter-clockwise, with $\mathcal{D}^{-}$mapped onto $\mathcal{D}_{*}^{+}$inside $C^{*}$ and $\mathcal{D}^{+}$onto $\mathcal{D}_{*}^{-}$outside $C^{*}$ ) so that

$$
\begin{aligned}
(2 \pi \mathrm{i}) J^{-}[F(z)] & =\oint_{C^{*}} F\left(\frac{1}{\tau}\right) \frac{\zeta \mathrm{d} \tau}{(\tau-\zeta) \tau} \\
& =\oint_{C^{*}} F\left(\frac{1}{\tau}\right)\left(\frac{1}{\tau-\zeta}-\frac{1}{\tau}\right) \mathrm{d} \tau=\oint_{C^{*}} \frac{F(1 / \tau)}{\tau-\zeta} \mathrm{d} \tau,
\end{aligned}
$$

in which $\oint_{C^{*}} F(1 / \tau) \mathrm{d} \tau / \tau=2 \pi \mathrm{i} F(\infty)=0$ due to $|F(z)|<$ $O\left(|z|^{-2}\right)$ as $|z| \rightarrow \infty$, resulting, by Eq. (1), in Eq. (14), and $J^{-}[F(z)] \rightarrow 0$ as $|z| \rightarrow \infty$. In this analogy, we obtain

Theorem 7. Integral formulas for complement function $F(z)$. The functional $J^{-}[F(z)]$ and its derivatives $J_{n}^{-}[F(z)]$ of a complement function $F(z)$ satisfy the integral formulas $(\forall n=0,1, \cdots)$

$$
\begin{aligned}
J_{n}^{-}[F(z)] & \equiv \frac{1}{2 \pi \mathrm{i}} \oint_{C^{-}} \frac{F^{(n)}(t)}{t-z} \mathrm{~d} t \\
& =\frac{-1}{2 \pi \mathrm{i}} \oint_{C} \frac{F^{(n)}(t)}{t-z} \mathrm{~d} t=F^{(n)}(z), \quad\left(z \in \mathcal{D}^{-}\right), \\
J_{n}^{-}[F(z)] & \equiv \frac{1}{2 \pi \mathrm{i}} \oint_{C^{-}} \frac{F^{(n)}(t)}{t-z} \mathrm{~d} t \\
& =\frac{-1}{2 \pi \mathrm{i}} \oint_{C} \frac{F^{(n)}(t)}{t-z} \mathrm{~d} t=0, \quad\left(z \in \mathcal{D}^{+}\right) .
\end{aligned}
$$

In sum, we note that the values of $J_{n}[f(z)]$ and $J_{n}^{-}[F(z)]$ are now given in the entire $z$-plane for all $n=0,1, \cdots$ in entirety, including $\forall z \in C$, since by analogy with Eqs. (6a)(6c), we also have:

Theorem 8. Uniform continuity of complement function $F(z)$ and its derivatives $F^{(n)}(z)$ in closed domain $\overline{\mathcal{D}^{-}}$and uniform convergence of their integral formulas. If $F(z)$ satisfies Eq. (14a) $\forall z \in \mathcal{D}^{-}$, and $E q$. (14b) $\forall z \in \mathcal{D}^{+}$, then $F(z)$ and its derivatives $F^{(n)}(z)$ are uniformly continuous in $\overline{\mathcal{D}^{-}}$, and their 
integral formulas are uniformly convergent in analogy to that for $f(z)$,

$$
\begin{aligned}
F^{(n)-}(z) & =F^{(n)}(z) ; \\
F^{(n)}(z) & =\frac{1}{\pi \mathrm{i}} \mathcal{P} \oint_{C^{-}} \frac{F^{(n)}(t)}{t-z} \mathrm{~d} t \quad(z \text { on } C), \\
& =\frac{-1}{\pi \mathrm{i}} \mathcal{P} \oint_{C} \frac{F^{(n)}(t)}{t-z} \mathrm{~d} t,
\end{aligned}
$$

for $n=0,1, \cdots$, where $F^{(n)-}(z)$ is the limit of $F^{(n)}(z)$ as $z$ reaches $C$ from the $\mathcal{D}^{-}$side.

Regarding uniform continuity and uniform convergence, Theorems 3 and 4 for $f(z)$ and Theorem 8 for $F(z)$ are of fundamental importance because to them all the general theorems on uniform continuity and uniform convergence known in calculus then hold. The six limiting equations in Eqs. (6c), (12) and (16) thus set the foundation established here for further developments, some to follow next.

\subsection{Integrals of the integral formulas}

In this respect, we have

Theorem 9. Integral theorem of functionals $J_{n}[f(z)]$ and $J_{n}^{-}[F(z)]$. The contour integrals of $J_{n}[f(z)]$ defined by Eq. (11) and that of $J_{n}^{-}[F(z)]$ by Eq. (15) along contour $C$ all vanish,

$$
\begin{aligned}
& \oint_{C} J_{n}[f(z)] \mathrm{d} z=\oint_{C} f^{(n)}(z) \mathrm{d} z=0, \\
& \oint_{C} J_{n}^{-}[F(z)] \mathrm{d} z=\oint_{C} F^{(n)}(z) \mathrm{d} z=0,
\end{aligned}
$$

In fact, the first follows from the integral formula (3), the uniform convergence relation (8) and the uniform continuity of $f^{(n)}(z)$ within closed domain $\overline{\mathcal{D}^{+}}$, and the second relation by analogy.

Aside from functionals $J_{n}[f(z)]$ and $J_{n}^{-}[F(z)]$, there are also functionals $K_{n}[f(z)]$ and $K_{n}^{-}[F(z)]$, for $n=0,1, \cdots$, with $z$ strictly located on contour $C$, i.e.

$$
\begin{aligned}
& K_{n}[f(z)] \equiv \frac{1}{\pi \mathrm{i}} \mathcal{P} \oint_{C} \frac{f^{(n)}(t)}{t-z} \mathrm{~d} t=f^{(n)}(z), \\
& K_{n}^{-}[F(z)] \equiv \frac{-1}{\pi \mathrm{i}} \mathcal{P} \oint_{C} \frac{F^{(n)}(t)}{t-z} \mathrm{~d} t=F^{(n)}(z),
\end{aligned}
$$

Theorem 10. Integral theorem of $K_{n}[f(z)]$ and $K_{n}^{-}[F(z)]$. The contour integrals of functionals $K_{n}[f(z)]$ and $K_{n}^{-}[F(z)]$ of Eq. (18a) along contour $C$ all vanish, for $n=0,1, \cdots$,

$$
\begin{aligned}
& \oint_{C} K_{n}[f(z)] \mathrm{d} z=\oint_{C} f^{(n)}(z) \mathrm{d} z=0, \\
& \oint_{C} K_{n}^{-}[F(z)] \mathrm{d} z=\oint_{C} F^{(n)}(z) \mathrm{d} z=0,
\end{aligned}
$$

This can be shown by analogy with Eq. (17), or by a direct proof with $z$ strictly located on contour $C$.

\section{Mean value of regular functions}

Formulas (1) and (11) provide a mean value of $f(z)$ and $f^{(n)}(z)$ for a circular contour in particular. The discussions here will be addressed only on $f(z)$, for the corresponding results for complement function $F(z)$ can be implied by analogy.

Theorem 11. Mean-value theorem. If each of $f^{(n)}(z)$ exists and is continuous inside and on a circular contour $C$, $t-z=r \mathrm{e}^{\mathrm{i} \theta}$ (of arbitrary radius $r$ ), formulas (1a) and (11a) become

$f^{(n)}(z)=\frac{1}{2 \pi} \int_{0}^{2 \pi} f^{(n)}\left(z+r \mathrm{e}^{\mathrm{i} \theta}\right) \mathrm{d} \theta, \quad(n=0,1,2, \cdots)$,

asserting that the value of a regular function $f^{(n)}(z)$ at the center of a circle $C$ is equal to the mean of its values on $C$. As $r \rightarrow 0$, Eq. (19), being homogeneous in $f^{(n)}$, becomes an identity.

In general, let $\left|f^{(m)}(t)\right| \leq M_{m}(R, z)$ be the upper bound on $|t-z|=R$, then by Eq. (10a),

$\left|f^{(n)}(z)\right| \leq(n-m) ! R^{-(n-m)} M_{m}(R, z)$,

$(R=|t-z|, m=0,1, \cdots n ; n=0,1,2, \cdots)$.

To explore dependence of $M_{m}(R, z)$ on $R=|t-z|$ and $z$, let us first consider a class of function $f(z)$ that possesses a Taylor series $f(z)=\Sigma_{n=0} c_{n} z^{n}$, convergent absolutely inside a circle $|z|=R$ lying within $\mathcal{D}^{+}$. Let $|f(z)| \leq M=\max _{|z|=R}|f(z)|$ for $|z| \leq R$, then

$\left|c_{n}\right| R^{n} \leq M(R), \quad$ (Cauchy's inequality),

which in turn provides for $\left|f^{(n)}(0)\right|$, in virtue of $f^{(n)}(0)=n ! c_{n}$, with the upper bounds

$\left|f^{(n)}(0)\right| \leq n ! R^{-n} M(R), \quad(n=0,1,2, \cdots)$.

As a proof for formulas (21) and (22), we deduce from Eq. (9a), for $|t|=R$, the relation

$\left|f^{(n)}(0)\right| \leq \frac{n !}{2 \pi} \oint_{C} \frac{M}{R^{n+1}}|\mathrm{~d} t|=n ! R^{-n} M(R)$,

in agreement with relation (20) for $z=0$ and $m=0$. An immediate consequence of relation (22) is the following.

Theorem 12. Liouville's theorem. If $f(z)$ is analytic and bounded for all finite $z$, it is a constant.

In fact, if $|f(z)| \leq M$ for all $z,\left|f^{(n)}(0)\right| \rightarrow 0$ as $R=|z| \rightarrow \infty$ by relation (22) for all $n \geq 1$, leaving only the $n=0$ term to give $f(z)=c_{0}$, a constant. By an extension in scope, we have

Theorem 13. Polynomial theorem. If $f^{(m)}(z)$ is analytic and $\left|f^{(m)}(z)\right| \leq M_{m}($ const. $>0) \forall z, f(z)$ is a polynomial of degree $m$.

First, by Liouville's theorem, $f^{(m)}(z)$ is a constant, hence by relation (20), $\left|f^{(n)}(0)\right| \rightarrow 0$ as $R \rightarrow \infty \forall n \geq m+1$, 
as was to prove. This theorem may be called the extended Liouville's theorem.

\section{The generalized Hilbert transforms}

We next pursue whether there exists an integral analog of the Cauchy-Riemann differential relations (2) between the conjugate functions $u$ and $v$ of an analytic function $f(z)=$ $u(x, y)+\mathrm{i} v(x, y)$. This leads to Hilbert's integral transform we now discuss.

\subsection{The Hilbert transform}

We first consider a class of analytic function $f(z)$ which is regular in the upper half $z$-plane for $\operatorname{Im} z \geq 0$, and vanishes as $|z| \rightarrow \infty$ uniformly in $0 \leq \arg z \leq \pi$; then by formula (1) for this $f(z)$ we take $C$ along the upper semicircular contour $C_{u}=C_{x}(-R \leq x \leq R)+C_{R}^{+}\left(C_{R}^{+}: z=R \mathrm{e}^{\mathrm{i} \theta}, R=\right.$ const., $0 \leq \theta \leq \pi)$, the integral on $C_{R}^{+} \rightarrow 0$ as $R \rightarrow \infty$, giving, by formula (1),

$\oint_{C_{u}} \frac{f(z)}{z-\zeta} \mathrm{d} z=\int_{-\infty}^{\infty} \frac{f(x)}{x-\zeta} \mathrm{d} x=2 \pi \mathrm{i} f(\zeta), \quad(\operatorname{Im} \zeta>0)$,

$\oint_{C_{u}} \frac{f(z)}{z-\zeta} \mathrm{d} z=\int_{-\infty}^{\infty} \frac{f(x)}{x-\zeta} \mathrm{d} x=0, \quad(\operatorname{Im} \zeta<0)$.

The limit of this set of equations as $\zeta \rightarrow \xi$ (a point on the real $\zeta$-axis), from above or from below, has been obtained for arbitrary contour in relation (6c) which can be adapted to the present geometry to give

$$
\begin{aligned}
& f^{+}(\xi)=f(\xi) ; \\
& f(\xi)=\frac{1}{\pi \mathrm{i}} \mathcal{P} \int_{-\infty}^{\infty} \frac{f(x) \mathrm{d} x}{x-\xi}, \quad(-\infty<\xi<\infty) .
\end{aligned}
$$

This shows that by Theorem $3, f(z)$ is uniformly continuous in the closed domain $\overline{\mathcal{D}_{u}^{+}}:(0 \leq|z| \leq R<\infty, 0 \leq \arg z \leq \pi$, or $y \geq 0)$. Hence substituting $f(x)=u(x)+\mathrm{i} v(x), f(\xi)=$ $u(\xi)+\mathrm{i} v(\xi)$ in Eq. Eq. (23), with $(u, v) \in C^{1}(-\infty<x<\infty)$ being understood, yields for the real and imaginary parts as

$$
\begin{aligned}
& u(\xi)=H[v(x)]=\frac{1}{\pi} \mathcal{P} \int_{-\infty}^{\infty} \frac{v(x) \mathrm{d} x}{x-\xi}, \\
& v(x)=H^{-1}[u(\xi)]=\frac{-1}{\pi} \mathcal{P} \int_{-\infty}^{\infty} \frac{u(\xi) \mathrm{d} \xi}{\xi-x} .
\end{aligned}
$$

This pair of reciprocal integral relations, known as the Hilbert transform, is due to David Hilbert (1862-1943), with $H$ denoting the transform and $H^{-1}$ the inverse transform, here based on Eq. (23) as their proof. In relations (24), $u(x)$ is said to be conjugate to $v(x)$; the relationship is skewreciprocal, with $v(x)$ conjugate to $-u(x)$, reciprocal with a minus sign. For a function regular for $\operatorname{Im} z \geq 0$, e.g. $f(z)=\mathrm{e}^{\mathrm{i} z}, \mathrm{e}^{\mathrm{i} x}=\cos x+\mathrm{i} \sin x$, we have $\cos \xi=H[\sin x]$, and by inversion, $\sin (x)=H^{-1}[\cos \xi]=-H[\cos \xi]$, in skew reciprocity. Now, a symbolic substitutions of the two opera- tor equations (24) gives

$$
\begin{gathered}
H^{-1} H[v(x)]=v(x), \quad H H^{-1}[u(\xi)]=u(\xi), \\
\longrightarrow \quad H^{-1} H=H H^{-1}=1, \quad \text { (unity operator) }
\end{gathered}
$$

This can be shown for specific $u(x)$ or $v(x)$ by consecutive evaluations of the integrals as exemplified here, whereas showing this for arbitrary $u(x)$ or $v(x)$ involves interchanging the order of integrations involving product of two Cauchy kernels. For the general case, it is essential to have

\section{The Poincaré-Bertrand formula}

$$
\begin{aligned}
\int_{L} \frac{\mathrm{d} t^{\prime}}{t^{\prime}-x} \int_{L} \frac{f\left(t, t^{\prime}\right)}{t-t^{\prime}} \mathrm{d} t= & \int_{L} \mathrm{~d} t \int_{L} \frac{f\left(t, t^{\prime}\right) \mathrm{d} t^{\prime}}{\left(t^{\prime}-x\right)\left(t-t^{\prime}\right)} \\
& -\pi^{2} f(x, x), \quad(x \in L),
\end{aligned}
$$

where $L$ is a regular Jordan arc, assumed finite (or infinite) in length, with end-points at $t=a$ and $t=b$ and without double point, the integration variable $t$ moves from $a$ to $b$, and function $f\left(t, t^{\prime}\right)$ is assumed regular in a neighborhood of the entire line $L$, while each of the integrals assumes its own principal value, here with the symbol $\mathcal{P}$ omitted as understood. For its proof we refer to a remark following Eq. (36) (or Muskhelishvili [6]) while we can illustrate here its application to relation (25).

Example 1. Consider the formula $H^{-1} H[v(x)]=v(x)$ for arbitrary $v(x)$ being operated by the Hilbert transform and its inversion in succession so that

$$
\begin{aligned}
H^{-1} H[v(x)] & =\frac{-1}{\pi^{2}} \int_{-\infty}^{\infty} \frac{\mathrm{d} t^{\prime}}{t^{\prime}-x} \int_{-\infty}^{\infty} \frac{v(t) \mathrm{d} t}{t-t^{\prime}} \\
& =v(x)+\frac{1}{\pi^{2}} \int_{-\infty}^{\infty} \frac{v(t) \mathrm{d} t}{x-t} \int_{-\infty}^{\infty}\left(\frac{1}{t^{\prime}-x}-\frac{1}{t^{\prime}-t}\right) \mathrm{d} t^{\prime} \\
& =v(x),
\end{aligned}
$$

where the second equality is due to the Poincaré-Bertrand formula and the last integral vanishes since $\mathcal{P} \int \mathrm{d} t^{\prime} /\left(t^{\prime}-x\right)=$ 0 . Similarly, we can show that $H H^{-1}[u(x)]=u(x)$ to complete a proof of relation (25).

Indeed, this also shows that when the two key relations in relation (6c) holds for a function $f(z)=u(x, y)+\mathrm{i} v(x, y)$, regular in a certain domain, to result in a skew-reciprocal pair of integral transform equations between its conjugate functions $u$ and $v$ (like that in the present case for the upper-half $z$-plane and three more to follow), the transform relations arrive automatically, without any need to prove $u$ and $v$ being conjugate functions (as in Titchmarsh(1948) [7]), because the proof is already imbedded in relation (6c). Also owing to relation (25), we point out that if $u(\xi)=H[v(x)]$ is regarded as a singular integral equation for $v(x)$ with $u(\xi)$ given (being Hilbert transformable), its solution is $v(x)=H^{-1}[u(\xi)]$, and vice versa.

In this case, if the Hilbert transform $u(\xi)=H[v(x)]$ of a transformable $v(x)$ has yielded the $u(\xi)$ to form a complex function $f(x)=u(x)+\mathrm{i} v(x)$ for $x$ real and have it analytically 
continued into $f(z)$ over the entire $z$-plane, then, by implication of the analysis underlying Eq. (23), $f(z)$ will be analytic and regular in the upper half $z$-plane, and, by Theorem 3 , be uniformly continuous in the closed domain $\overline{\mathcal{D}_{u}^{+}}$, while necessarily possessing, due to Liouville's Theorem, some singularities in the lower-half $z$-plane.

Example 2. Given $v(x)=\cos x$, then by Eq. (24), $u(\xi)=H[\cos x]=-\sin \xi$, so that $f(x)=u(x)+\mathrm{i} v(x)=$ $\mathrm{i}(\cos x+\mathrm{i} \sin x)=\mathrm{ie}^{\mathrm{i} x}$, hence $f(z)=\mathrm{ie}^{\mathrm{i} z}$, being regular for $y \geq 0$, but singular as $y \rightarrow-\infty$.

\subsection{The complementary Hilbert transform}

On the contrary, if $F(z)$ is regular in the lower half $z$-plane, then we have $F(z)$, regular in $\mathcal{D}^{-}\{z \mid \operatorname{Im} z \leq 0\}$, satisfying, by Eq. (16), the relations

$$
\begin{aligned}
& F^{-}(\xi)=F(\xi) ; \\
& F(\xi)=\frac{-1}{\pi \mathrm{i}} \mathcal{P} \int_{-\infty}^{\infty} \frac{F(x) \mathrm{d} x}{x-\xi}, \quad(-\infty<\xi<\infty),
\end{aligned}
$$

where $F^{-}(\xi)$ is the limit of $F(z)$ as a point $z(\operatorname{Im} z<0)$ tends from below to reach a point $\xi$ on the real $z$-axis, so that relation (27) differs from the corresponding relation (23) only by a minus sign of the integral by virtue of Eq. (15). Thus, substituting $F(x)=U(x)+\mathrm{i} V(x)$ and $F(\xi)=U(\xi)+\mathrm{i} V(\xi)$ in relation (27) yields

$$
\begin{aligned}
& U(\xi)=\bar{H}[V(x)]=\frac{-1}{\pi} \mathcal{P} \int_{-\infty}^{\infty} \frac{V(x) \mathrm{d} x}{x-\xi}, \\
& V(x)=\bar{H}^{-1}[U(\xi)]=\frac{1}{\pi} \mathcal{P} \int_{-\infty}^{\infty} \frac{U(\xi) \mathrm{d} \xi}{\xi-x}, \\
& \longrightarrow \quad \bar{H}[V(x)]=-H[V(x)]=H^{-1}[V(x)], \\
& \bar{H}^{-1}[U(\xi)]=H[U(\xi)]=-H^{-1}[U(\xi)] .
\end{aligned}
$$

The pair of reciprocal integral relations in Eq. (28a), designated by $\bar{H}[\cdot]$ and its inverse by $\bar{H}^{-1}[\cdot]$, may be called the complementary Hilbert transform; it is related to the Hilbert transform by Eq. (28b).

Example 3. Given $v(x)=-\left(x^{2}+1\right)^{-1}$, its Hilbert transform is found, after some algebra, as

$$
\begin{aligned}
& u(\xi)=\frac{-1}{\pi} \mathcal{P} \int_{-\infty}^{\infty} \frac{\mathrm{d} x}{\left(x^{2}+1\right)(x-\xi)}=\frac{\xi}{\xi^{2}+1}, \\
& f(x)=u(x)+\mathrm{i} v(x)=\frac{1}{x+\mathrm{i}} \rightarrow f(z)=\frac{1}{z+\mathrm{i}},
\end{aligned}
$$

which is regular in the upper half $z$-plane, but has a simple pole at $z=-\mathrm{i}$ in the lower half $z$-plane. On the other hand, the complementary transform of $V(x)=-\left(x^{2}+1\right)^{-1}$ gives, by Eq. (28a),

$U(\xi)=\bar{H}[V(x)]=-H[V(x)]=\frac{-\xi}{\xi^{2}+1}$

$$
\begin{aligned}
& \rightarrow \quad F(x)=U+\mathrm{i} V=\frac{-1}{x-\mathrm{i}} \\
& \rightarrow \quad F(z)=\frac{-1}{z-\mathrm{i}},
\end{aligned}
$$

which is regular in the lower half $z$-plane, but has a simple pole at $z=\mathrm{i}$ in the upper half $z$-plane.

Thus, a given $v(x)$ can generate an analytic function $f(z)$ (or $F(z)$ ) which is regular in the upper (or lower) half $z$-plane by applying the Hilbert (or complementary Hilbert) transform.

Example 4. For $V(x)=\cos x$, we have, by Eq. (28), $U(\xi)=\sin \xi$, hence $F(x)=\mathrm{i}(\cos x-\mathrm{i} \sin x)=\mathrm{ie}^{-\mathrm{i} x}$ and hence $F(z)=\mathrm{ie}^{-\mathrm{i} z}$, which behaves opposite to $f(z)=\mathrm{ie}^{\mathrm{i} z}$ of Example 2.

\subsection{The circular Hilbert transform}

We next consider a function $f(z)$ which is regular in open domain $\mathcal{D}_{c}^{+}$of a unit disc: $|z|<1$ and continuous on $|z|=1$ for the contour $C$ which contains both points $z=\mathrm{e}^{\mathrm{i} \theta}$ and $t=\mathrm{e}^{1 \phi}$ to give, by the general formula (6c), for $(-\pi \leq \theta \leq \pi)$,

$$
\begin{aligned}
f\left(\mathrm{e}^{\mathrm{i} \theta}\right) & =\frac{1}{\pi} \mathcal{P} \int_{-\pi}^{\pi} f\left(\mathrm{e}^{1 \phi}\right) \frac{\mathrm{e}^{\mathrm{i} \phi} \mathrm{d} \phi}{\mathrm{e}^{\mathrm{i} \phi}-\mathrm{e}^{\mathrm{i} \theta}} \\
& =\frac{1}{2 \pi} \mathcal{P} \int_{-\pi}^{\pi}\left(1-\mathrm{i} \cot \frac{\theta-\phi}{2}\right) f\left(\mathrm{e}^{1 \phi}\right) \mathrm{d} \phi, \\
u(\theta) & =\frac{\mathcal{P}}{2 \pi} \int_{-\pi}^{\pi}\left(u(\phi)+v(\phi) \cot \frac{\phi-\theta}{2}\right) \mathrm{d} \phi, \\
v(\phi) & =\frac{\mathcal{P}}{2 \pi} \int_{-\pi}^{\pi}\left(v(\theta)-u(\theta) \cot \frac{\theta-\phi}{2}\right) \mathrm{d} \theta,
\end{aligned}
$$

which results from separating the real and imaginary parts in Eq. (29a) with $f\left(\mathrm{e}^{\mathrm{i} \theta}\right) \equiv \hat{f}(\theta)=u(\theta)+\mathrm{i} v(\theta)$. This pair of integral transform relations are self-consistent, and can be used as is. Finally, we have

Theorem 14. Circular integral transform. If $f(z)$ is regular in $|z| \leq 1$, and if $f(0)=0$, then

$$
\begin{gathered}
f(0)=\int_{-\pi}^{\pi} f\left(\mathrm{e}^{\mathrm{i} \theta}\right) \mathrm{d} \theta=\int_{-\pi}^{\pi}\{u(\theta)+\mathrm{i} v(\theta)\} \mathrm{d} \theta=0, \\
u(\theta)=\hat{H}[v(\phi)]=\frac{1}{2 \pi} \int_{-\pi}^{\pi} v(\phi) \cot \frac{\phi-\theta}{2} \mathrm{~d} \phi, \\
v(\phi)=\hat{H}^{-1}[u(\theta)]=\frac{-1}{2 \pi} \int_{-\pi}^{\pi} u(\theta) \cot \frac{\theta-\phi}{2} \mathrm{~d} \theta .
\end{gathered}
$$

Here, Eq. (30a) is implied by the mean-value theorem (19), hence Eq. (29b) reduces (by suppressing the value $f(0)$, if $f(0) \neq 0$ ) to the ultimate form Eq. (30b). In fact, Eq. (29) and Eq. (30) can serve to resolve discrepancies between some similar yet differing published results, all called Hilbert's reciprocity formula for the cotangent-kernel (e.g. Magnus \& Oberhettinger [8], Muskhelishvili [6]). This pair of Eq. (30b) will be called the circular Hilbert transform. 


\subsection{The complementary circular transform}

In analogy with the Hilbert and its complementary transforms, we can also deduce the transform for the class of function $F(z)$ which is regular in domain $\mathcal{D}_{c}^{-}\{\forall z:|z| \geq 1(F(z) \rightarrow$ 0 as $z \rightarrow \infty)\}$. For $F(z)$, we simply take Eq. (30) with a change in sign of the integrals, as implied by Eqs. (15)-(16), giving for $F\left(\mathrm{e}^{\mathrm{i} \theta}\right) \equiv \breve{F}(\theta)=U(\theta)+\mathrm{i} V(\theta)$ the transform equations as

$$
\begin{aligned}
& U(\theta)=\check{H}[V(\phi)]=\frac{-1}{2 \pi} \int_{-\pi}^{\pi} V(\phi) \cot \frac{\phi-\theta}{2} \mathrm{~d} \phi, \\
& V(\phi)=\check{H}^{-1}[U(\theta)]=\frac{1}{2 \pi} \int_{-\pi}^{\pi} U(\theta) \cot \frac{\theta-\phi}{2} \mathrm{~d} \theta,
\end{aligned}
$$

while $\hat{H}[\cdot]$ and $\check{H}[\cdot]$ are analogous with $\hat{H}[\cdot]$ in Eq. (30a) standing for $H[\cdot]$ in Eq. (24) and with $\breve{H}[\cdot]$ in Eq. (31) for $\bar{H}[\cdot]$ in Eq. (28). The above pair of relations will be called the complementary circular transform.

Example 5. As a simple example, we take $v(\phi)=\sin \phi$, then its circular transform, by Eq. (30), is

$u(\theta)=\frac{1}{2 \pi} \mathcal{P} \int_{-\pi}^{\pi} \sin (\psi+\theta) \frac{1+\cos \psi}{\sin \psi} \mathrm{d} \psi=\cos \theta$,

giving $f\left(\mathrm{e}^{\mathrm{i} \theta}\right)=u(\theta)+\mathrm{i} v(\theta)=\mathrm{e}^{\mathrm{i} \theta}$, and hence its analytically continued function $f(z)=r \mathrm{e}^{\mathrm{i} \theta}=z$, which is regular in $\mathcal{D}_{c}^{+}$, with $f(0)=0$, but is singular at infinity in $\mathcal{D}_{c}^{-}$. On the other hand, for $V(\phi)=\sin \phi$, we take the complementary circular transform equation (31), then $U(\theta)=-u(\theta)=-\cos \theta$, giving $F\left(\mathrm{e}^{\mathrm{i} \theta}\right)=U(\theta)+\mathrm{i} V(\theta)=-\mathrm{e}^{-\mathrm{i} \theta}$, and therefore $F(z)=$ $-\left(r \mathrm{e}^{\mathrm{i} \theta}\right)^{-1}=-z^{-1}$, which is regular inside $\overline{\mathcal{D}_{c}^{-}}:(|z| \geq 1)$, with a simple zero at $z=\infty$, but has a simple pole at $z=0$ in $\mathcal{D}_{c}^{+}$, as is implied by Theorem 8 or Liouville's Theorem.

\subsection{The Parseval relations for the generalized Hilbert transforms}

If $u(x)$ and $v(x)$ of Eq. (24) are both square integrable, and similarly for their complementary counterpart, $U(x), V(x)$ of Eq. (28), and further for the pairs $u(\theta), v(\theta)$ of Eq. (30) and $U(\theta), V(\theta)$ of Eq. (31), they satisfy the Parseval relations

$$
\begin{aligned}
& \int_{-\infty}^{\infty} u^{2}(x) \mathrm{d} x=\int_{-\infty}^{\infty} v^{2}(x) \mathrm{d} x, \\
& \int_{-\infty}^{\infty} U^{2}(x) \mathrm{d} x=\int_{-\infty}^{\infty} V^{2}(x) \mathrm{d} x, \\
& \int_{-\pi}^{\pi} u^{2}(\theta) \mathrm{d} \theta=\int_{-\pi}^{\pi} v^{2}(\phi) \mathrm{d} \phi, \\
& \int_{-\pi}^{\pi} U^{2}(\theta) \mathrm{d} \theta=\int_{-\pi}^{\pi} V^{2}(\phi) \mathrm{d} \phi .
\end{aligned}
$$

Proof: For the first Parseval relation, we have $u(x)=H[v(t)]$, $v(t)=H^{-1}[u(x)]$, then, by Eq. (24)

$$
\int_{-\infty}^{\infty} u^{2}(x) \mathrm{d} x=\frac{1}{\pi} \int_{-\infty}^{\infty} u(x) \mathrm{d} x \int_{-\infty}^{\infty} \frac{v(t) \mathrm{d} t}{t-x}
$$

$$
\begin{aligned}
& =\frac{-1}{\pi} \int_{-\infty}^{\infty} v(t) \mathrm{d} t \int_{-\infty}^{\infty} \frac{u(x) \mathrm{d} x}{x-t} \\
& =\int_{-\infty}^{\infty} v^{2}(t) \mathrm{d} t,
\end{aligned}
$$

by interchanging the order of integration. Similarly, the other Parseval relations can be proved.

Here, we point out that these four generalized integral transforms all stem from the keystone relations (6c) which can be attributed to have provided the foundation on which these integral transforms are given their proof.

\section{Behavior of Cauchy function $f(z)$ in the complemen- tary domain $\mathcal{D}^{-}$}

We have seen exemplified in Examples 2-5 that while a Cauchy function $f(z)$ is regular in a closed domain $\overline{\mathcal{D}^{+}}$, it invariably has one or more singularities in its complementary domain $\mathcal{D}^{-}$. In general, if $f(z)$ is a Cauchy function, regular inside and on contour $C$, then $f(z)$ is implied by Liouville's theorem to possess a singularity distribution in $\mathcal{D}^{-}$ outside $C$, including $z=\infty$, unless $f(t) \equiv A$ (const.) $\forall t \in C$. Our primary objective here is to exhibit the exact relationship between the singularities of $f(z)$ in $\mathcal{D}^{-}$and their values $f(t) \forall t \in C$ by illustrating some typical direct problems.

In the direct problem, the singularities of $f(z)$ are first prescribed explicitly in $\mathcal{D}^{-}$outside $C$ in order to examine their corresponding integral formulas on $C$, which in this section will be a unit circle $|z|=1$ for simplicity. Let one such singularity be located at $z_{1}$ outside $C$, which duly induces a corresponding singularity at $z=\infty$, e.g. a pole (or a zero) at $z_{1}$ inducing a zero (or a pole) of the same order at $z=\infty$; an algebraic or a logarithmic branch point at $z_{1}$ being associated with the same branch at $z=\infty$, all to be accounted for. With all the singularities of $f(z)$ explicitly distributed in $\mathcal{D}^{-}$, its values on $C$ are trivially $f(z)=f(t) \forall|t|=1$. Inversely, any $f(t)$ given analytically on $C$ gives the singularity in $f(z)$. In general practice, $f(t)$ is known only numerically on $C$.

Example 6. In Eq. (1a), given $f(t)=(t-a)^{-1}(|a|>1)$, a simple pole situated at $t=a$ outside $C$, hence, by Eq. (1a), $J[f(z)]=f(z)=(z-a)^{-1}$, which is regular for $|z| \leq 1 \in \mathcal{D}^{+}$, whereas for $|z|>1$,

$$
\begin{aligned}
2 \pi \mathrm{i} J[f(z)] & =\oint_{C} g(t, z) \mathrm{d} t=I_{C}=0, \\
(g(t, z) & \left.=(t-a)^{-1}(t-z)^{-1},|a|>1,|z|>1\right), \\
2 \pi \mathrm{i} J[f(z)] & =I_{\infty}-I_{S}=\left\{\oint_{C_{\infty}}-\oint_{C_{S}}\right\} g(t, z) \mathrm{d} t \\
& =\frac{1}{z-a} \oint_{C_{S}}\left(\frac{1}{t-a}-\frac{1}{t-z}\right) \mathrm{d} t=0,
\end{aligned}
$$

where integral $I_{C}$ is on contour $C(|t|=1), I_{\infty}$ on $C_{\infty}$ encircling $t=\infty$, and $I_{S}$ enclosing both poles of $g(t, z)$ (all in the positive sense), and they are related by Eq. (3b) with 
$I_{\infty}=I_{C}+I_{S}$, since $g(t, z)$ is regular in the domain bounded by $C_{\infty}, C$ and $C_{S}$. Separately, $I_{C}=0$ by Eq. (3b) $(g(t, z)$ being regular $\forall|t| \leq 1,|a|>1,|z|>1), I_{\infty}=0$ since $g(t, z)=\left(t^{-2}+O\left(|t|^{-3}\right)\right)$ with residue res. $=0$ at $z=\infty$, and $I_{S}=0$ since the residues of its integrand at $t=a(r e s .=1)$ and at $t=z($ res. $=-1)$ cancel. Whence $J[f(z)]=0$ for $|z|>1$ is shown both by applying Eq. (3b) and alternatively by three direct integrations.

Example 7. As a variation, let the complement function in Eq. (14a) be $F(t)=t^{-n}(n=1,2, \cdots)$ which is a pole of or$\operatorname{der} n$ at $t=0$ and is regular for $|t| \geq 1$, then for $|z| \geq 1$ we have $J^{-}[F(z)]=I_{C^{-}}=(2 \pi \mathrm{i})^{-1} \oint_{C^{-}} t^{-n} /(t-z) \mathrm{d} t=z^{-n}$ by Eq. (14a), or by direct evaluation, $I_{\infty}=I_{S}+I_{C}=0$ (due to its zero residue at $t=\infty$ ), hence $I_{C^{-}}=-I_{C}=I_{S}=z^{-n}$ (the residue at $t=z,|z|>1)$. For $|z|<1$,

$$
\begin{gathered}
2 \pi \mathrm{i} J^{-}[F(z)]=\oint_{C^{-}} g(t, z) \mathrm{d} t=I_{C^{-}}=0, \\
\left(g(t, z)=t^{-n}(t-z)^{-1},|z|<1\right), \\
2 \pi \mathrm{i} J^{-}[F(z)]=I_{C^{-}}=-I_{C}=-I_{\infty}=0,
\end{gathered}
$$

where the first result for $I_{C^{-}}=0(|z|<1)$ is by Eq. (15b) of Theorem 7, whereas the alternative second results from direct integration by deforming the contour $C$ to $C_{\infty}$, between which $g(t, z)$ is regular and $g(t, z)=O\left(|t|^{-(n+1)}\right)$ with zero residue at $t=\infty$, hence giving both in agreement.

Example 8. In Eq. (1a), given $f(t)=(t-1 / b)^{-1 / 2}(0<b<$ $1)$, a branch made single-valued on a two-sheet Riemann surface cut along the real $t$-axis from branch point at $t=1 / b>1$ to $t=+\infty$, hence by Eq. (1a), we have $J[f(z)]=(z-1 / b)^{-1 / 2}$ which is regular for $|z| \leq 1(1 / b>1)$, whereas for $|z|>1$,

$$
\begin{aligned}
2 \pi \mathrm{i} J[f(z)] & =\oint_{C} g(t, z) \mathrm{d} t=I_{C}=0, \\
(g(t, z) & \left.=(t-1 / b)^{-1 / 2}(t-z)^{-1},|z|>1\right), \\
2 \pi \mathrm{i} J[f(z)] & =\oint_{C_{I}} g\left(\frac{1}{\xi}, z\right) \frac{\mathrm{d} \xi}{\xi^{2}} \\
& =\frac{2 \sqrt{b}}{z}\left\{\int_{0}^{b} \frac{\mathrm{d} \xi}{\sqrt{\xi(b-\xi)}(1 / z-\xi)}-\frac{\pi z}{\sqrt{1-b z}}\right\} \\
& =0, \quad(1<|z|<1 / b),
\end{aligned}
$$

where $I_{C}=0$ by Eq. (3b), $\xi=1 / t$ is the inverse mapping, with contour $C_{I}(|\xi|=1)$ in the positive sense, the line integral from $\xi=0$ to $b$ comes from the contour integral around the cut within $C_{I}$, whilst the last term in the bracket comes with the residue at $\xi=1 / z$ (located within $C_{I}$ ). We note that the value $I_{C}=0$ (or equivalently, $\oint_{C_{o}} g(t, z) \mathrm{d} t=0$ ) is necessary and sufficient to have the line integral determined as shown above. A similar result can be attained when point $z$ falls on the branch cut.

Example 9. Finally, let us consider the special case with $f(t) \equiv 1$ on $|t|=1$, for which we have

$$
\begin{aligned}
J[f(z)] & =\frac{1}{2 \pi \mathrm{i}} \oint_{C} \frac{\mathrm{d} t}{t-z}=I_{C}=1, \quad(|z| \leq 1), \\
J[f(z)] & =I_{\infty}-I_{S} \\
& =\frac{1}{2 \pi \mathrm{i}} \oint_{C_{\infty}}\left(\frac{1}{t}+O\left(|t|^{-2}\right)\right) \mathrm{d} t-\frac{1}{2 \pi \mathrm{i}} \oint_{C_{S}} \frac{\mathrm{d} t}{t-z} \\
& =1-1=0, \quad(|z|>1),
\end{aligned}
$$

where integral $I_{\infty}$ is on $C_{\infty}, I_{S}$ is on contour $S(|t|=1)$ encircling $t=z(|z|>1)$. Therefore, even with $J[f(z)] \equiv 1(|z| \leq 1)$, the functional $J[f(z)]$ still jumps down to $J[f(z)] \equiv 0(\forall z$ : $|z|>1$ ), hence the integral formula (1b) invoking $J[f(z)] \equiv 0$ for $z \in \mathcal{D}^{-}$is universally fulfilled. It is also evident that the functional $J[f(z)]$ is not a function, for it is neither continuous nor differentiable in a neighborhood striding across contour $C$. The only exception for $J[f(z)]$ to be continuous over the entire $z$-plane is when $f(t) \equiv 0$ on $C$.

\section{The Plemelj formulas.}

We now consider another general class of line integrals of the form

$f(z)=\frac{1}{2 \pi \mathrm{i}} \int_{L} \frac{g(t)}{t-z} \mathrm{~d} t, \quad(z \notin L)$,

where $L$ is a regular Jordan arc, assumed finite (or infinite) in length, with end-points at $t=a$ and $t=b$ and without double point, and with the integration moving from $a$ to $b$ (see Fig. 2), and $g(t)$ is assumed regular in a neighborhood of the entire line $L$. Evidently, $f(z)$ is a regular function $\forall z \notin L$ and has a simple zero at infinity. It is also evident that $f(z)$ possesses derivatives to all orders, given by

$$
f^{(n)}(z)=\frac{n !}{2 \pi \mathrm{i}} \int_{L} \frac{g(t) \mathrm{d} t}{(t-z)^{n+1}}, \quad(z \notin L, n=1,2, \cdots) .
$$
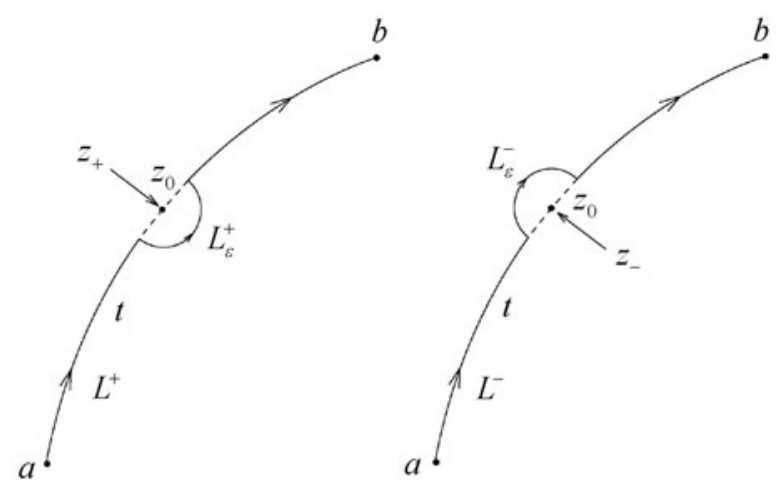

Fig. 2 A Plemelj integral $f(z)=(2 \pi \mathrm{i})^{-1} \int_{L} g(t) \mathrm{d} t /(t-z)$ along an open path $L$ (from $t=a$ to $t=b$ ) has its path $L$ deformed into $L^{ \pm}$ with only an indented small semicircle $L_{\epsilon}^{ \pm}\left(\left|t-z_{0}\right|=\epsilon\right)$, on the Five side of $L$, centered at $z_{0} \in L$ so as to let a point $z$ on the \pm ive side (left/right side) of $L$ tend to $z_{0} \in L$ without crossing $L^{ \pm}$ 
In the limit as point $z$ tends to a point $t=z_{0}$ on $L$ from the left (+ive), or from the right (-ive) side of $L$, we indent $L$ into $L^{ \pm}=L_{\epsilon}^{ \pm}+L_{p}$ where $L_{\epsilon}^{ \pm}$is a semicircle of radius $\left|t-z_{0}\right|=\epsilon$ onto the (干ive) side, leaving $L_{p}=L^{ \pm}-L_{\epsilon}^{ \pm}$intact, so that point $z$ reaches $z_{0} \in L$ without crossing $L^{ \pm}$, while $f(z)$ tends to its limit $f^{+}(z)$, or $f^{-}(z)$, respectively, yet undetermined. Carrying out the integration over $L^{ \pm}$paths in a way similar to that for $C^{ \pm}$in Sect. 3, we obtain Plemelj's formula

$f^{ \pm}(z)= \pm \frac{1}{2} g(z)+\frac{1}{2 \pi \mathrm{i}} \mathcal{P} \int_{L} \frac{g(t)}{t-z} \mathrm{~d} t$,

$$
\text { ( } z \in L \text { - Plemelj's formula), }
$$

with $z_{0}$ becoming generic $z$, the $\operatorname{sign} \mathcal{P}$ (often omitted) signifying its Cauchy principal value, hence

$$
\begin{aligned}
& f^{+}(z)-f^{-}(z)=g(z), \quad(z \in L), \\
& f^{+}(z)+f^{-}(z)=\frac{1}{\pi \mathrm{i}} \mathcal{P} \int_{L} \frac{g(t)}{t-z} \mathrm{~d} t, \quad(z \in L) .
\end{aligned}
$$

Formulas (35a)-(35c) are called Plemelj's formulas. Finally, substituting Eq. (35b) in Eq. (33) yields

$f(z)=\frac{1}{2 \pi \mathrm{i}} \int_{L} \frac{g(t)}{t-z} \mathrm{~d} t=\frac{1}{2 \pi \mathrm{i}} \int_{L} \frac{f^{+}(t)-f^{-}(t)}{t-z} \mathrm{~d} t$,

which shows that $f(z)$ is determined by its jump $\left[f^{+}(t)-\right.$ $\left.f^{-}(t)\right]$ across the line $L$ for all $z$ in the plane, including the $z$ 's on $L$, on which Eq. (36) reduces to an identity by virtue of Eqs. (35b)-(35c).

In applying Plemelj's formulas to integrals involving two Cauchy kernels, as exemplified in Example 1, it is essential to make use of the Poincaré-Bertrand formula (26). This formula can now be proved by letting a point $z \notin L$ tend to a point $z_{0} \in L$, while applying Plemelj's formula twice as needed. Plemelj's formulas can be powerful in treating various two-dimensional mathematical physics, including problems with mixed boundary conditions on harmonic and biharmonic functions, the Riemann-Hilbert problems and other types of problems encountered in science, technology and applications.

\section{Application and physical significance}

Applications of the formulas obtained in this study can be made to various scientific fields as well as for further mathematical advances. Here we first select a Riemann-Hilbert problem of aerodynamic wing theory for an exact solution to a two-dimensional flat plate airfoil. It involves dealing with a pair of conjugate integrals, one of which is about a finite Hilbert transform and its inversion [8], whilst the other a Plemelj's integral over an infinite line.

Thus we consider the 2-D irrotational flow of an incompressible and inviscid fluid past a flat plate airfoil held fixed along $-1 \leq x \leq 1, y=0$ in an inertial frame of reference at an incidence angle $\alpha$ with respect to a uniform free stream of velocity $\boldsymbol{U}$. Denoting the fluid velocity by $(U \cos \alpha+u, U \sin \alpha+v),(u, v)$ being the perturbation velocity, we have the basic equations (cf. Kármán \& Burgers [9]) as

$u_{x}+v_{y}=0, \quad$ (incompressibility),

$u_{y}-v_{x}=0, \quad$ (irrotationality),

$v=-U \sin \alpha, \quad(-1 \leq x \leq 1, y= \pm 0)$;

$u^{2}+v^{2} \rightarrow 0, \quad\left(\right.$ as $\left.x^{2}+y^{2} \rightarrow \infty\right)$,

$\frac{p}{\rho}+\frac{1}{2}\left\{(U \cos \alpha+u)^{2}+(U \sin \alpha+v)^{2}\right\}=\frac{1}{2} U^{2}$.

Here Eqs. (37a) and (37b) are the 2-D components of $\nabla \cdot \boldsymbol{u}=0$ and $\nabla \times \boldsymbol{u}=0$, respectively, $\boldsymbol{u}$ being the vector $(u, v, 0)$. With Eq. (37c) providing the boundary conditions, a solution to $(u, v)$ can be found from Eqs. (37a)-(37c), and Eq. (37d) then gives the Bernoulli equation for pressure $p$ in the fluid of density $\rho$.

Noting that $(u,-v)$ satisfy the Cauchy-Riemann equations (37a) and (37b), the complex velocity $w=u-\mathrm{i} v$ is then an analytical function of $z=x+\mathrm{i} y$. Since $v(x, y)$ is prescribed in Eq. (37c) as being even in $y$, then $u(x, y)$ by Eqs. (37a) and (37b) is odd in $y$. Since $w(z)$ is analytic and regular in the open domain of the flow field, hence $u(x, 0)=0$ for $|x|>1$ since $u(x, y)$ is there continuous and odd in $y$, rendering this a Riemann-Hilbert problem. It has a complementary solution, which is $w_{c}=u_{c}-\mathrm{i} v_{c}=\mathrm{i} / H(z), H(z)=\sqrt{z^{2}-1}$, for on $y=$ $0, H^{ \pm}(x)= \pm \mathrm{i} \sqrt{1-x^{2}}(|x|<1)$ and $H^{ \pm}(x)=\operatorname{sgn} x \sqrt{x^{2}-1}$ $(|x|>1)$, so that $v_{c}=0$ for $|x|<1, u_{c}=0$ for $|x|>1$, and $\left|w_{c}\right| \rightarrow 0$ as $|z| \rightarrow \infty$. Now introducing $f(z)=w(z) H(z)$ gives

$$
\begin{gathered}
f^{+}(x)-f^{-}(x)=2 \sqrt{1-x^{2}} v(x), \\
(|x|<1, v(x)=-U \sin \alpha), \\
f^{+}(x)-f^{-}(x)=0, \quad(|x|>1) .
\end{gathered}
$$

Hence, by Plemelj's formula (36) (here with the path $L$ spanning the entire $x$-axis), we have

$$
w(z)=\frac{1}{\pi \mathrm{i} \sqrt{z^{2}-1}} \int_{-1}^{1} \frac{\sqrt{1-t^{2}}}{t-z} v(t) \mathrm{d} t+\frac{\mathrm{i} B}{\sqrt{z^{2}-1}},
$$

$B$ being an arbitrary real constant to qualify $w(z)$ as a solution with $|w(z)|=O\left(|z|^{-1}\right)$ as $z \rightarrow \infty$ and $w(z)$ being integrable at the plate as required. Finally, $B$ is determined by an additional physical condition, known as Kutta's condition, requiring Ref. [9] that $w(z)$ be regular in a neighborhood of the trailing edge at $z=1$. Hence expanding this $w(z)$ about $z=1$ yields under Kutta's condition the unique exact solution as

$$
\begin{aligned}
w(z)= & -\frac{1}{\pi \mathrm{i}} \sqrt{\frac{z-1}{z+1}} \int_{-1}^{1} \sqrt{\frac{1+t}{1-t}} \frac{v(t)}{t-z} \mathrm{~d} t, \\
(0 & \leq|z|<\infty),
\end{aligned}
$$

valid for arbitrary $v(x)$. For the flat plate, $v(x)=-U \sin \alpha$ by Eqs. (37c), (38) reduces by using Eq. (35a) to 


$$
\begin{gathered}
u^{ \pm}(x)-\mathrm{i} v^{ \pm}(x)=U \sin \alpha\left( \pm \sqrt{\frac{1-x}{1+x}}+\mathrm{i}\right), \\
(-1<x \leq 1),
\end{gathered}
$$

exhibiting that $u$ has an equal and opposite jump across the plate by a distribution having a square root singularity at the leading edge at $z=-1$ and vanishing at the trailing edge at $z=1$. This jump distribution of $u$ results in the so-called circulation, $\Gamma$, around the plate (see, e.g. von Kármám \& Burgers [9]), given by the contour integral of $u$ clockwise (by convention) around the airfoil,

$\Gamma=\oint u(x, \pm 0) \mathrm{d} x=2 \pi U \sin \alpha$.

Finally, expressed in three-dimensional vectors, $\boldsymbol{U}=$ ( $U \cos \alpha, U \sin \alpha, 0), \boldsymbol{\Gamma}=(0,0,-\Gamma)$ (by the right-hand rule with the contour integral for $\Gamma)$, and $\boldsymbol{L}=\left(L_{1}, L_{2}, 0\right)$ for the lift vector acting on the airfoil, we have lift $\boldsymbol{L}$ given by the Kutta-Joukowski theorem [9] in vector cross product of $\boldsymbol{U} \times \boldsymbol{\Gamma}$ as

$\boldsymbol{L}=\rho \boldsymbol{U} \times \boldsymbol{\Gamma}, \quad L=|\boldsymbol{L}|=2 \pi \rho U^{2} \sin \alpha$,

by which the lift $\boldsymbol{L}$ acts perpendicular to the free stream velocity $\boldsymbol{U}$, pointing upward if positive.

Regarding this problem and extension in scope, there are several issues for expository study.

\subsection{Physical significance and advances in applications}

Mathematically, the above solution to the airfoil problem as formulated is exact. It can serve as a standard reference for assessing approximate approaches such as by linear theory for small incidence angles. It can further set a methodology useful for achieving exact solutions in extended scope. In the latter aspect, we first note that physically, the square root singularity of velocity at the leading edge (associated with an even worse singular suction in pressure $p$ by Eq. (37d)) should raise serious questions concerning not only for engineering applications but further for experimental verification of the range of validity of the theory. Such concerns have actually stimulated innovative theoretical developments by adding a distribution of flow-mass sources along the plate to obtain, again in exact form, real airfoil profiles enclosing the lifting flat plate and its singularity (lying inside the airfoil now having a round nose at the leading edge and a cusped trailing edge). This exact theory can then be subjected to specific engineering design and wind tunnel tests for validation and adaptation by the industry. In return, the result is gratifying that some airfoils with aptly designed round noses have been found experimentally capable of sustaining the low suction pressure for incidence angle $\alpha$ up to around 18 degrees before the airfoils stall, so to speak, with flow separation due eventually to the fluid viscous effects. This approach to determine accurate solutions to problems of fluid flow past bodies of finite volume by placing flow singularities at an ultimate focal point or plane (e.g. at the center of a circle or sphere or at the focal ellipse of a tri-axial ellipsoid) is now classical for mechanics and electrodynamics, followed by more advanced methods for aerodynamics of thin airfoils (e.g Lighthill [10]), for naval hydrodynamics of double-body for ship hull design (e.g. Wu \& Chwang [11]), for biharmonics of triaxial ellipsoids and in other cases. Returning to the mathematics, the infinite suction pressure acting at the pointed leading edge can indeed be integrated with rigor to produce a so-called finite leading edge suction $S$ pulling the airfoil forward along the flat plate just so exactly as to make the resultant lift $\boldsymbol{L}$ (as the vector sum of the singular leadingedge suction force $\boldsymbol{S}$ and the pressure integral acting normal to the plate) to act exactly normal to the free stream velocity $\boldsymbol{U}$, as predicted by the Kutta-Joukowski theorem and well supported by experiments. In this respect, having the exact solution can provide a concrete foundation for further advanced developments.

\subsection{Finite Hilbert transform and its inversion}

When Cauchy integrals enter the analysis of physical problems, the primary query would be on the significance of the Cauchy kernel $(t-z)^{-1}$. In mathematical physics, Cauchy integrals are closely related to the potentials of single and double layers distributed along a contour $C$ or an open $\operatorname{arc} L$, as we can illustrate next. For this airfoil problem, there are actually alternative approaches for the solution. We have elected above to regard it as a Riemann-Hilbert problem. But we can also represent the flat plate in the free stream by a distribution of flow singularities fixed to the plate by the so-called singularity method. For 2-D flows satisfying Eqs. (37a) and (37b), there are basic elementary flow singularities known as a point source of strength $Q$ and a point vortex of strength $\Gamma$ held at the origin, say, inducing their flow velocities at a field point $z$ as

$$
\begin{gathered}
w(z)=u(x, y)-\mathrm{i} v(x, y)=\frac{Q+\mathrm{i} \Gamma}{2 \pi z} \\
\longrightarrow \overline{w(z)}=u+\mathrm{i} v=\frac{Q-\mathrm{i} \Gamma}{2 \pi r} \mathrm{e}^{\mathrm{i} \theta},
\end{gathered}
$$

which, now expressed in the polar coordinates, $z=x+\mathrm{i} y=$ $r \mathrm{e}^{\mathrm{i} \theta}$, shows that source $Q$ has only an outward radial velocity component $u_{r}=Q / 2 \pi r(\arg \bar{w}=\theta=$ const. $)$, and vortex $\Gamma$ has only a clockwise circumferential velocity component $u_{\theta}=-\Gamma / 2 \pi r(\arg \bar{w}=\theta-\pi / 2)$. In terms of these base singularities, we can construct a surface distribution of sources of density $q$ and a vortex sheet of density $\gamma$ per unit length along a regular $\operatorname{arc} L$, generating a complex velocity field $w(z)$ as

$w(z)=\frac{1}{2 \pi} \int_{L} \frac{q(t)+\mathrm{i} \gamma(t)}{z-t} \mathrm{~d} t$,

here with the Cauchy kernel physically signified. For the flat plate airfoil held fixed in a free stream, we need only a surface distribution of vortex sheet along the plate, giving its complex velocity as 
$w(z)=\frac{1}{2 \pi \mathrm{i}} \int_{-1}^{1} \frac{\gamma(t)}{t-z} \mathrm{~d} t$.

From this we have, by Plemelj's formula (35), that on the \pm sides of the plate,

$$
\begin{aligned}
w^{ \pm}(x) & =u^{ \pm}(x)-\mathrm{i} v^{ \pm}(x) \\
& = \pm \frac{1}{2} \gamma(x)+\frac{1}{2 \pi \mathrm{i}} \int_{-1}^{1} \frac{\gamma(t)}{t-x} \mathrm{~d} t, \quad(|x|<1), \\
\longrightarrow \quad u^{+}(x)-u^{-}(x)=\gamma(x), \quad(|x|<1), & \\
v^{+}(x) & =v^{-}(x)=\frac{1}{2 \pi} \int_{-1}^{1} \frac{\gamma(t)}{t-x} \mathrm{~d} t \equiv G[\gamma(t)],
\end{aligned}
$$$$
(|x|<1) .
$$

With the boundary conditions (37c) prescribing $v(x)$, Eq. (44c) actually is a singular integral equation for the vorticity distribution $\gamma(x)$, which mathematically can also be regarded as a finite Hilbert transform, with the integral operator $G$ denoting the transform. Then its inversion can be given by Eq. (44b), with $u^{+}-u^{-}=\gamma(x)$ taken from Eq. (38) with its construction shown for arbitrary $v^{ \pm}(x)=v(x)$, thereby yielding the unique exact solution for a flat plate airfoil as

$$
\begin{aligned}
v(x) & =\frac{1}{2 \pi} \int_{-1}^{1} \frac{\gamma(t)}{t-x} \mathrm{~d} t \equiv G[\gamma(t)] \\
\longrightarrow & \gamma(x)=G^{-1}[v(t)], \quad(|x|<1), \\
\gamma(x) & =-\frac{2}{\pi} \sqrt{\frac{1-x}{1+x}} \int_{-1}^{1} \sqrt{\frac{1+t^{\prime}}{1-t^{\prime}}} \frac{v\left(t^{\prime}\right)}{t^{\prime}-x} \mathrm{~d} t^{\prime} \\
& \equiv G^{-1}\left[v\left(t^{\prime}\right)\right], \quad(|x|<1),
\end{aligned}
$$

where the integral operator $G$ defines the finite Hilbert transform and the operator $G^{-1}$ its inversion, signifying $G G^{-1}=$ $G^{-1} G=1$ (the unity operator). This can be verified by substituting Eq. (45b) into the integral equation (45a) with applying the Poincaré-Bertrand formula (26) as shown in Example 1.

\subsection{A fully nonlinear theory for a flexible wing traversing an arbitrary trajectory}

The foregoing primary development can afford a sound basis to develop a fully nonlinear theory for a two-dimensional flexible wing moving with arbitrary unsteady variations in wing profile and along arbitrary trajectory for modeling bird/insect flight and fish swimming. Kinematically, the irrotational flow of an incompressible and inviscid fluid is produced by a two-dimensional flexible lifting surface $S_{b}(t)$ of negligible thickness, stretched straight at time $t=0$ and moving with time $t>0$ through the fluid with time-varying wing shape along an arbitrary trajectory. The motion is prescribed by a coupled Lagrangian-Eulerian system with the Lagrangian body coordinates $(\xi, \eta)$ to identify a point $X(\xi, t), Y(\xi, t)$ on $\eta=0$ covering the wing surface $S_{b}(t)$ and the vortex sheet $S_{w}(t)$ shed from the wing, both of which can be prescribed by a complex coordinate $z=x+\mathrm{i} y$ (for the Eu- ler description) fixed in an absolute inertial frame (with fluid at rest far away), and with $z=Z(\xi, t)$ prescribing the unsteady body-wake motion function, parametrically in $\xi$ (with $\eta=0)$ as

$$
\begin{aligned}
& Z(\xi, t)=X(\xi, t)+\mathrm{i} Y(\xi, t), \\
& \quad \text { on } \quad S_{b}(t):(-1<\xi<1)+S_{w}(t):\left(1<\xi<\xi_{m}\right),
\end{aligned}
$$

with $\xi=-1$ at the leading and $\xi=1$ at the trailing edge of the wing. From the trailing edge a vortex sheet is being shed under Kutta' a condition to form a prolonging wake $S_{w}$, and $\xi_{m}$ identifies the path $Z\left(\xi_{m}, t\right)$ of the starting vortex shed at $t=0$ to reach $\xi_{m}=\xi_{m}(t)$ at time $t$. Starting from the initial condition with $Z(\xi, 0)=\xi(-1<\xi<1, \eta=0)$, the point $\xi$ on $S_{b}(t)$ moves for $t>0$ with a prescribed body motion function $Z(\xi, t)$ and a prescribed complex velocity $W(\xi, t)=U-\mathrm{i} V$ (see Fig. 3a),

$$
\begin{gathered}
W(\xi, t)=U-\mathrm{i} V=\partial \bar{Z} / \partial t=X_{t}-\mathrm{i} Y_{t}, \\
(|\xi|<1, t \geq 0 ; \bar{Z}=X-\mathrm{i} Y),
\end{gathered}
$$

which has a tangential component, $U_{s}(\xi, t)$, and a normal component, $U_{n}(\xi, t)$, given by

$W \partial Z / \partial \xi=\left(X_{\xi} X_{t}+Y_{\xi} Y_{t}\right)-\mathrm{i}\left(X_{\xi} Y_{t}-Y_{\xi} X_{t}\right)=U_{s}-\mathrm{i} U_{n},(46 \mathrm{c})$ on $S(t)=S_{b}(t)+S_{w}(t)$, the flexible $S_{b}(t)$ being assumed inextensible $(|\partial Z / \partial \xi|=1)$. Thus, the normal velocity, $U_{n}(\xi, t)$, is prescribed for $|\xi|<1$ while the wake vortex, once shed, is conserved as free vortex moving fixed, by Helmholtz's Theorem, with the local fluid velocity, which is a part of the solution.

For the wing motion, $S_{b}$ moves with a shape function $Z(\xi, t)$ consisting of (a) rectilinear translation with velocity $\partial Z_{0} / \partial t$ at incidence angle $\alpha(t)$, (b) rotation with angular velocity $\Omega(t)=-\mathrm{d} \theta / \mathrm{d} t, \theta=\arg (Z(1, t)-Z(-1, t))$, and (c) flexing with camber function $\hat{Z}(\xi, t)$ prescribed by (see Fig. 3b)

$$
\begin{aligned}
Z(\xi, t) & =Z_{0}(t)+\mathrm{e}^{\mathrm{i} \theta} \hat{Z}(\xi, t), \\
\hat{Z}(\xi, t) & =\hat{X}(\xi, t)+\mathrm{i} \hat{Y}(\xi, t)=\hat{X}(\xi, t)+\mathrm{i} F(\hat{X}(\xi, t), t),
\end{aligned}
$$

where $\hat{Z}(\xi, t)$ is the complex camber function given in the body frame, with the origin set at $Z_{0}(t)$, corresponding to $\hat{X}(0, t)=0, Z_{0}=Z(0, t)-\mathrm{ie}^{\mathrm{i} \theta} \hat{Y}(0, t)$, with $\hat{Y}(\xi, t)=$ $F(\hat{X}(\xi, t), t)$ being the real camber function, and with $|Z(1, t)-Z(-1, t)|=c(t)$ the chord length of the wing.

Now the problem is to determine the vorticity $\gamma(\xi, t)$ over $S(t)=S_{b}(t)+S_{w}(t)$. For the solution, the simple and elegant physical concept crystallized by von Kármán and Sears (1938) [12] has provided an ingenious restructuring of the vorticity distribution over $S(t)$ as follows

$$
\begin{aligned}
& \text { on } S_{b}(t): \gamma(\xi, t)=\gamma_{0}(\xi, t)+\gamma_{1}(\xi, t), \quad(-1<\xi<1), \\
& \text { on } S_{w}(t): \gamma(\xi, t)=\gamma_{w}(\xi, t), \quad\left(1<\xi<\xi_{m}\right) .
\end{aligned}
$$

Here, $\gamma_{0}(\xi, t)$ is the vortex bound to $S_{b}$ in the wakeless "quasi-steady" flow past $S_{b}$ based on steady airfoil theory to carry out the original assigned $U_{n}(\xi, t)$, with time $t$ frozen during each time step to serve as a parameter. Vorticity 
$\gamma_{1}(\xi, t)$ is the additional bound vortex induced on $S_{b}$ by the trailing wake vortex $\gamma_{w}(\xi, t)$, yet unknown, such that $\gamma_{1}$ and $\gamma_{w}$ make no change to $U_{n}$ over $S_{b}$ so that the original as- signed normal velocity $U_{n}(\xi, t)$ prescribed on $S_{b}(t)$ is thus ever reinstated at each step.

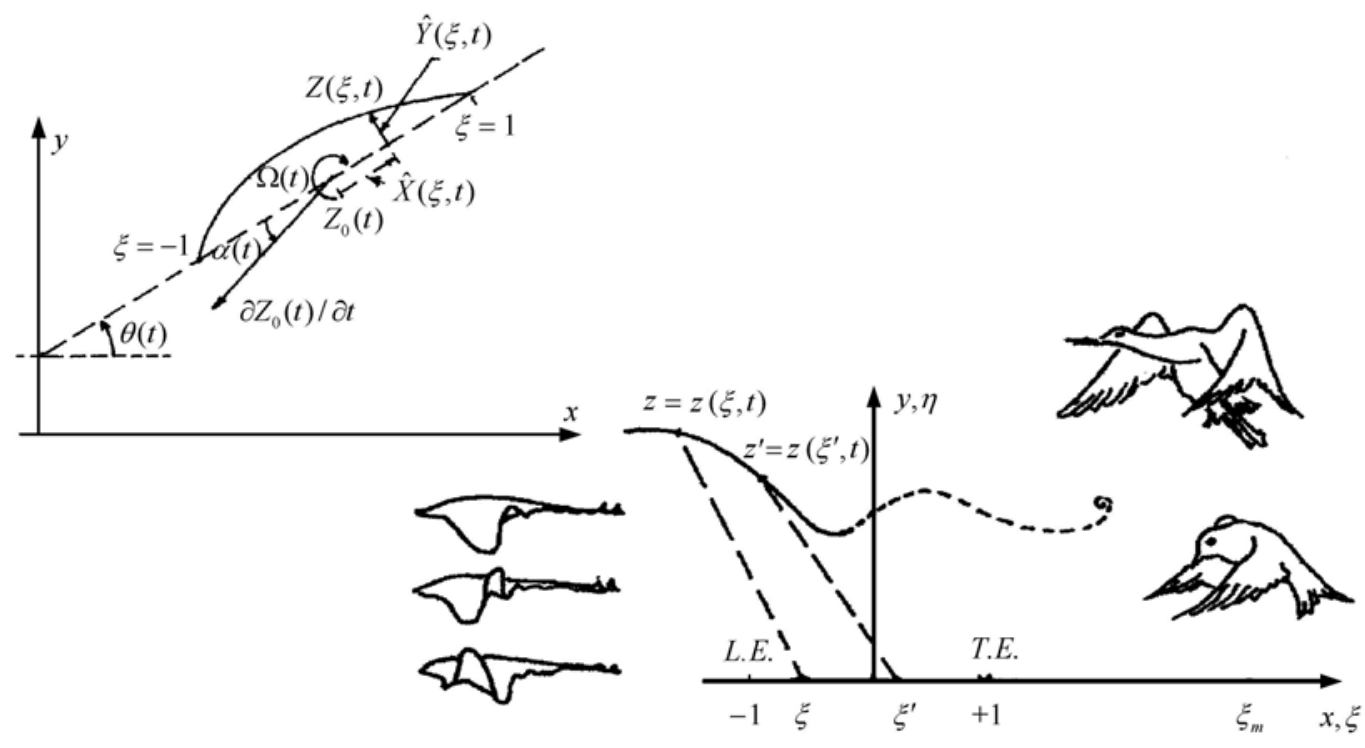

Fig. 3 The Lagrangian coordinates $(\xi, \eta)$ describe arbitrary motion of a 2-D flexible wing moving along arbitrary trajectory $\eta=0$ through unbounded fluid at rest at infinity: wing moves with (a) translational velocity $\left(\partial Z_{0} / \partial t\right)$ at incidence angle $\alpha(t)$, (b) rotational angular velocity $\Omega(t)$, and (c) unsteady camber function $\hat{Z}(\xi, t)$

Briefly, the initial quasi-steady vorticity $\gamma_{0}(\xi, 0+)$ opens a time-marching procedure with $t=t_{1}=\Delta t, t_{2}=2 \Delta t, \cdots(\Delta t$ taken aptly small). In the very first step, the shed vorticity element $\gamma_{w}\left(\Delta \xi, t_{1}\right)$ is related to $\gamma_{1}\left(\xi, t_{1}\right)(-1<\xi<$ 1 ), hence the solution at $t=t_{1}$ is obtained by applying Kelvin's Theorem on conservation of the total circulations (i.e. $\Gamma_{0}+\Gamma_{1}+\Gamma_{w}=\int_{-1}^{1}\left(\gamma_{0}+\gamma_{1}\right) \mathrm{d} \xi+\gamma_{w}\left(1, t_{1}\right) \Delta \xi=0$, $\Delta \xi=U \Delta t)$, then up-dated for each time step. This yields a nonlinear integral equation for the wake vorticity $\gamma_{w}$ as

$$
\begin{gathered}
\Gamma_{0}(t)+\int_{1}^{\xi_{m}}\left\{\sqrt{\frac{\xi+1}{\xi-1}}+N_{w}(\xi, t)+N_{b}(\xi, t)\right\} \gamma_{w}(\xi, t) \mathrm{d} \xi=0, \\
\left(\xi_{m}=\xi_{m}\left(t_{k}\right), k=1,2, \cdots\right),
\end{gathered}
$$

where $N_{b}(\xi, t)$ and $N_{w}(\xi, t)$ denote the nonlinear terms (pertaining separately to $S_{b}$ and $S_{w}$ ) (cf. Wu [13] Eq. (37)). This is proclaimed as the general wake-vorticity theorem in terms of this nonlinear integral equation, which is for wake vorticity $\gamma_{w}$ to satisfy exactly for the solution to be exact for a flexible wing varying in arbitrary wing geometry and its trajectory. In the linear limit, both $N_{w}$ and $N_{b}$ vanish, reducing Eq. (48) to Wagner's linear integral equation.

\subsection{Comparison between theory and experiments}

The nonlinear theory by $\mathrm{Wu}[13]$ has been applied by Stredie
(2005) [14] and Hou et al. (2007) [15] to carry out computations of various unsteady motions of a 2D airfoil to attain results of high accuracy and special interest over a diversified broad scope. Of them two special computational studies are presented here for comparisons with two well noted wind-tunnel experiments. The first is for an airfoil performing heaving oscillations at reduced frequency $\sigma=\omega c / U$ ( $\omega$ being the circular frequency, $c$ the half-chord, $U$ the free stream velocity) for the one-parameter family solution parametric in $\sigma$ and computed for $\sigma=2.0$ and heaving amplitude $h=0.038$ as that used in the experiment by Lai (2002) [16]. The numerical results (for time step $\Delta t=0.001$ to 2630 steps) is shown in Fig. 4, with the central photo depicting the observed wake vortices shed from the trailing edge situated near the left border, growing in size with their senses indicated on the top, and with the corresponding numerical result shown in equal scale right below with a single vortex line as the centerline of the real physically diffusing vortex wake. The qualitative and quantitative agreement between theory and experiment is excellent. More specifically, the distance between the centers of the second pair of vortices is measured in the photo to be very nearly 0.4 chord, so is exactly with the computed result. Figure $4 \mathrm{~b}$ is a computed result of the longitudinal fluid velocity, in excess of $U$, produced by the heaving wing at a point 0.41 chord downstream from the trailing edge. The negative values (for fluid flowing 
upstream) in the first half period gives a preciously interesting computed plot of the local velocity field during the shedding of the very first starting vortex, then followed by peri- odic fluctuations of the local wake velocity, which is largely positive, thus implying a forward thrust exerted by the fluid on the heaving wing.
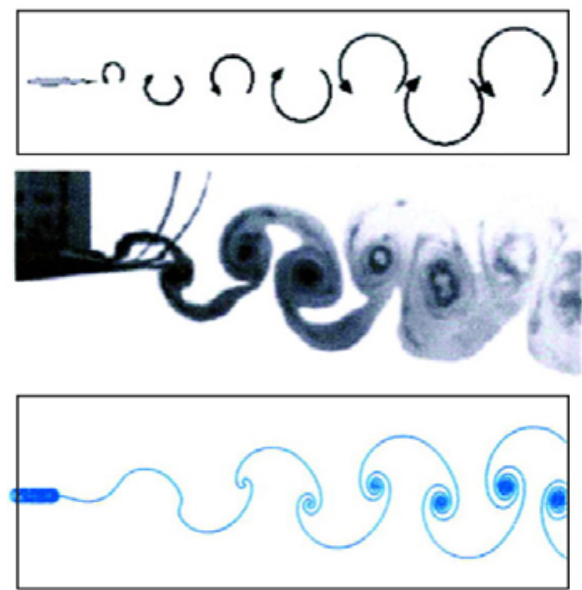

b

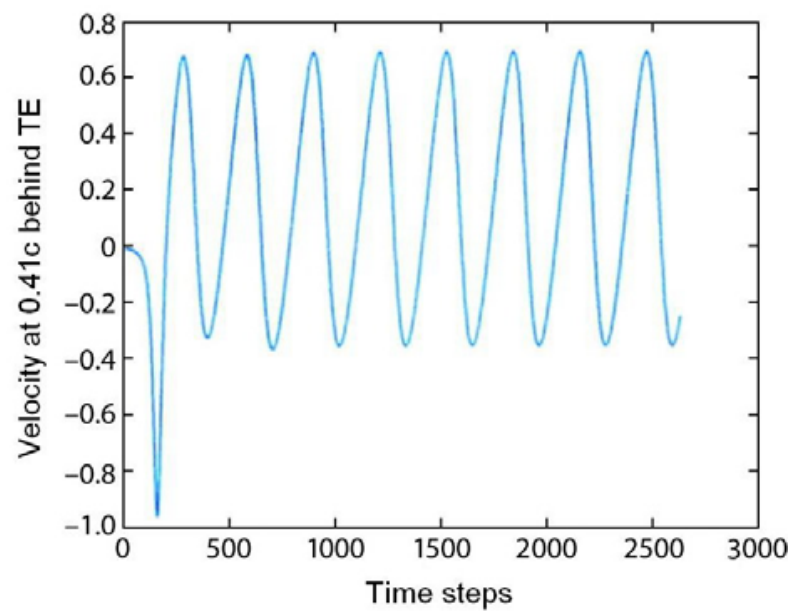

Fig. 4 a Heaving wing; Upper panel: rotational sense of eddies; Mid panel: experiment by Lai (2002); Lower panel: Wu's numerical results; $\mathbf{b}$ The flow velocity just downstream of a heaving wing, evaluated at 0.41 chord downstream of the trailing edge

The other case is for an airfoil performing pitching oscillations, about the quarter chord (or about the mid-cord plus heaving) computed with those data used in the experiment for the pitching amplitude of $\alpha=2^{\circ}$ with time step $\Delta t=0.0005$ to 3000 steps. Figure 5 shows the comparison of the numerical results with the experiment accomplished by Kooschefahani (1989) [17] in which the photo of the wake vortices shed from the wing (moving from left to right) is shown below the corresponding numerical result. The comparison is again excellent in precision. Both comparisons stress the utmost importance in having the very first starting vortex computed in the first time step in high accuracy, for a small artificial error is found to lead to growing departure of

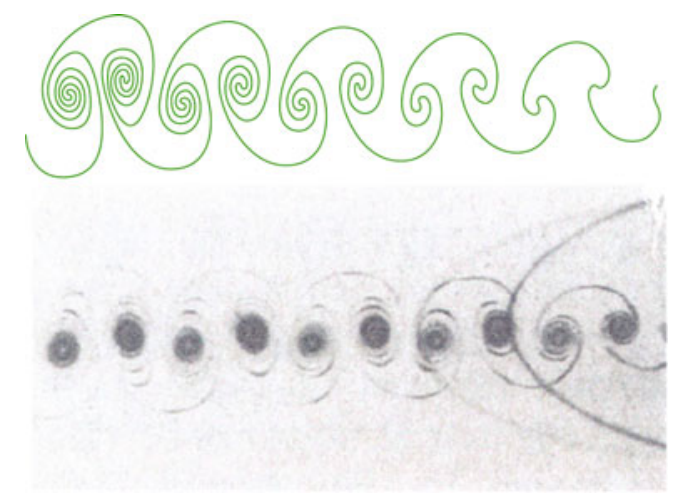

Fig. 5 A wing pitching about the 1/4-chord point and moving from left to right; Upper panel: Wu's numerical results; Lower panel: experiment by Koochesfahani (1989) the wake vortices, in both position and entire properties from the accurate results. This also holds for more cases examined, including mixed heave and pitch, time-periodic bending of the wing plate for simulating membrane wing (like that in bat flight), impulsive start in incidence and camber of a Fourier flexible wing, etc., as shown by Hou et al. (2007) [15]. The nonlinear unsteady theory of a 2D flexible wing has been extended by Hou et al. (2006) [18] to 3D for further computational studies, to which we refer for details.

\section{Discussion and conclusion}

The primary objective of the present study is first to extend the coverage of Cauchy's integral formulas (1a) and (1b) to include the point $z$ on contour $C$ of the integral and render it valid for the entire $z$-plane. With the generalized function $f(z)$ assumed $C^{n} \forall z \in \mathcal{D}^{+}$within $C$ and about $C, f(z)$ and all its derivatives $f^{(n)}(z)$ are proved to be uniformly continuous in the closed domain $\overline{\mathcal{D}^{+}}=\left[\mathcal{D}^{+}+C\right]$. Consequently, Cauchy's integral formulas (for $\left.J_{n}[f(z)], n=0,1, \cdots\right) \forall z \in$ $\mathcal{D}^{+}$(or $\forall z \in \mathcal{D}^{-}$) are proved uniformly convergent in closed domain $\forall z \in \overline{\mathcal{D}^{+}}$(or $\forall z \in \overline{\mathcal{D}^{-}}=\left[\mathcal{D}^{-}+C\right]$ ). These results of fundamental value can be ascribed to principle (1) in making indentation of $C$ possible and to principle (2) in assuring the validity of the results, both under the new assumption to produce the two key relations in relation $(6 c)$ that form the foundation of the present study. These new formulas have furnished a simple and sound base to establish the generalized Hilbert-type integral transforms in various domains of 
different geometry, which are adopted jointly with Plemelj's formulas in applications to engineering science, mathematical physics, and applied mathematics. In conclusion, there remain several vital issues of great significance for exposition.

\subsection{Overall behavior of the generalized function in the entire $z$-plane}

The foregoing deliberation on the overall properties of the generalized function $f(z)$ over the entire $z$-plane is comprehensively expounded by considering the direct problem. This has led to exhibiting the constitution of the inverse problem as demonstrated by Examples 6-9. In general practice, however, solutions to mathematical problems encountered in science and technology are found possibly involving certain analytical relations and conditions, e.g. the Bernoulli equation $(37 \mathrm{~d})$ for the airfoil problem, which is also a pressure condition used in studies on water waves with unknown surface displacements. In such cases, prevailing analytic relations can afford extended scope for functional analysis to give further data bases than the simplified version of the direct problem covered here.

\subsection{Relationship between the Cauchy integral formulas and Plemelj's formulas}

The sharp contrast between the overall behavior of Cauchy function $f(z)$ and its contour integral $J[f(z)]$ in Eq. (1) and the Plemelj integral equation (33) of $f(z)$ along a regular Jordan $\operatorname{arc} L$ without a double point (not closed as a contour) can be brought to focus as follows. Once when the two ends of Plemelj's integral path $L$ are brought to coincide to form a closed simple contour $C$ enclosing an open domain $\mathcal{D}^{+}$ bounded by $C$ and excluding an open domain $\mathcal{D}^{-}$outside $C$, then immediately the jumps of the Cauchy functional $J[f(z)]$ across contour $C$ and the jumps of $f^{ \pm}$across the Plemelj integral path $L$ suffer a drastic change in values of the integrals from one to the other. While the changes are valid mathematically in exact rigor, it may still seem in need for special attention. For instance, the two sets of infinite line integrals in Eqs. (24) and (28) are actually the limits of two distinct contours.

\subsection{A conjecture on the inverse problem}

Finally, we conclude this study with presenting an inverse problem of great significance as follows. This inverse problem is motivated to determine analytically all the singularity distributions of the generalized function $f(z)$ in the open domain $\mathcal{D}^{-}$outside contour $C$ in terms of its continuous values prescribed only numerically within and on $C$. The need is vastly broad in scope and long in history. A longstanding problem is relevant to drawing a conclusion on the convergence, or not, of the power series solution (started by Stokes [2] in 1847) for water waves in permanent form of all heights. Similar interests in having the inverse problem resolved also have inspired studies on perturbation expansion of solitary waves, e.g. by Wu et al. [4].

\section{The inverse problem.}

The inverse problem is to adopt the values of $f(t)$ prescribed only in numerics $\forall t$ on contour $C$ for a function $f(z)$, analytic and regular inside $C$, to determine analytically all the exact singularity distributions of $f(z) \forall z \in \mathcal{D}^{-}$outside $C$, whatever the singularity distribution.

The conjecture. Solution to this inverse problem is conjectured to exist.

Having a general methodology to this grand cause is of vital importance, for in studies of physical phenomena, solutions are usually found in numerics, so to have this inverse problem resolved is essential to gaining in-depth comprehension of the phenomenon in pursuit.

Acknowledgement Warmest thanks are due from me to Prof. Jia-Chun Li for his interesting comments and encouragement on Preprint [1] that have led me to bring forth the present version for publication. I also wish to thank sincerely Prof. Thomas Y. Hou, Prof. Joe Keller, Prof. John C.K. Chu, and Prof. Lu Ting for interesting discussions, and especially Prof. Jin V. Zhang at The Hong Kong University for careful reading of the text and analysis. I am most appreciative for the gracious encouragement from Dr. Chinhua S. Wu, F. Bai-Yueh Wu, and the American-Chinese Scholarship Foundation.

\section{References}

$1 \mathrm{Wu}$, Th.Y.: On uniform continuity of Cauchy's function and uniform convergence of Cauchy's integral formula with applications. arXiv.org:0710.5790v2

2 Stokes, G.G.: On the theory of oscillary waves. Trans. Cambridge Phil. Soc. 8, 441-455 (1847); Mathematical and Physical Papers 1, 197-225. Cambridge Univ. Press, Cambridge (1880)

3 Wu, Th.Y., Wang, X., Qu, W.: On solitary waves. Part 2, A unified perturbation theory for higher-order waves. Acta Mech. Sin. 21, 515-530 (2005)

$4 \mathrm{Wu}$, Th.Y., Kao, J., Zhang, J.E.: A unified intrinsic functional expansion theory for solitary waves. Acta Mech. Sin. 21, 1-15 (2005)

5 Titchmarsh, E.C.: The Theory of Functions. Oxford Univ. Press, Oxford (1949)

6 Muskhelishvili, N.I.: Singular Integral Equation. P. Noordhoff N.V. Groningen, Holland (1953)

7 Titchmarsh, E.C.: The Theory of Fourier Integrals. Oxford Univ. Press, Oxford (1948)

8 Magnus, W., Oberhettinger, F.: Formulas and Theorems for the Special Functions of Mathematical Physics. Chelsea Pub., New York (1949)

9 von Kármán, Th., Burgers, J.M.: General aerodynamic theory-perfect fluids. In: Aerodynamic Theory, Division E, Vol. II, Durand W.F. ed. 308 (1943) 
10 Lighthill, M.J.: A new approach to thin aerofoil theory. Aeron. Quart. 3, 193-210 (1951)

$11 \mathrm{Wu}$, Th.Y., Chwang, A.T.: Double-body flow theory-a new look at the classical problem. In: Tenth Symp. on Naval Hydrodynamics. ONR 89-106 Dep't of the Navy, Washington DC (1974)

12 von Kármán, Th., von Sears, W.R.: Airfoil theory for nonuniform motion. J. Aero. Sci. 5, 379-390 (1938)

$13 \mathrm{Wu}$, Th.Y.: A nonlinear theory for unsteady flexible wing. J. Eng. Math. 58, 279-287 (2007)

14 Stredie, V.G.: Mathematical modeling and simulation of aquatic and aerial animal locomotion. [Ph.D. Thesis], Califor- nia Institute of Technology, Pasadena, CA (2005)

15 Hou, T.Y., Stredie, V.G., Wu, Th.Y.: A 3D numerical method for studying vortex formation behind a moving plate. Communication in Computational Physics 1, 207-228 (2006)

16 Lai, J.C.S., Yue, J., Platzer, L.F.: Control of backward-facing step flow using a flapping foil. Exp. Fluids 32, 44-54 (2002)

17 Kooschesfahani, M.M.: Vortical patterns in the wake of an oscillating airfoil. AIAA J. 27, 1200-1205 (1989)

18 Hou, T.Y., Stredie, V.G., Wu, T.Y.: Mathematical modeling and simulation of aquatic and aerial animal locomotion. J. Comput. Phys. 225, 1603-1631 (2007) 